University of Nebraska - Lincoln

DigitalCommons@University of Nebraska - Lincoln

Timothy J. Gay Publications

Research Papers in Physics and Astronomy

February 1992

\title{
Mott electron polarimetry
}

Timothy J. Gay

University of Nebraska - Lincoln, tgay1@unl.edu

F. B. Dunning

Rice Quantum Institute, Rice University, Houston, Texas

Follow this and additional works at: https://digitalcommons.unl.edu/physicsgay

Part of the Physics Commons

Gay, Timothy J. and Dunning, F. B., "Mott electron polarimetry" (1992). Timothy J. Gay Publications. 38. https://digitalcommons.unl.edu/physicsgay/38

This Article is brought to you for free and open access by the Research Papers in Physics and Astronomy at DigitalCommons@University of Nebraska - Lincoln. It has been accepted for inclusion in Timothy J. Gay Publications by an authorized administrator of DigitalCommons@University of Nebraska - Lincoln. 


\title{
Mott electron polarimetry
}

\author{
T. J. Gay \\ Department of Physics, University of Missouri-Rolla, Rolla, Missouri 65401 \\ F. B. Dunning \\ Department of Space Physics and Astronomy and the Rice Quantum Institute, Rice University, Houston, \\ Texas 77251
}

(Received 5 July 1991; accepted for publication 13 October 1991)

\begin{abstract}
Electron polarimeters based on Mott scattering are extensively used in atomic and molecular, solid state, nuclear, and high-energy physics. This use stems from the increasing realization that much additional information concerning many physical processes can be obtained through spin-dependent measurements. In this review we discuss the basic physics and application of Mott polarimetry. A number of different Mott polarimeter designs are described that illustrate the wide range of operating energies (10 eV-1 MeV) and geometries that can be used in such instruments. The calibration of Mott polarimeters is discussed together with the potential sources of systematic error that can arise and that can limit measurement accuracies. The aim is to present a comprehensive practical guide to Mott polarimetry and the capabilities of the technique.
\end{abstract}

\section{INTRODUCTION}

The idea that electrons have an intrinsic spin angular momentum and associated spin magnetic moment was introduced by Goudsmit and Uhlenbeck ${ }^{1}$ in 1925 to explain the fine structure of certain atomic spectral features. By 1927, electron spin had been successfully incorporated into the nonrelativistic quantum theory ${ }^{2}$ and, one year later, Dirac showed that it was an integral part of the relativistic wave theory of electrons. ${ }^{3}$ While the experimental evidence for electron spin was compelling, it was also indircet, rclying as it did on the study of electrons bound in atomic systems. In 1929 Mott raised the question as to whether effects due to electron spin could be observed directly. ${ }^{4,5}$ Using uncertainty principle arguments he attributed to Bohr, Mott pointed out the impossibility ${ }^{6}$ of measuring the spin magnetic moment directly or using it, in a SternGerlach-type experiment, to separate electrons of different spin. He proposed instead that the spin magnetic moment could be detected in a double scattering experiment schematically illustrated in Fig. 1 in which a beam of unpolarized electrons is initially scattered at high energies from high- $Z$ nuclei in a target beam or foil. Because of the socalled spin-orbit interaction, which will be discussed in the next section, large angle $\left(\theta_{1} \geqslant 90^{\circ}\right)$ scattering from the first target produces electrons with a significant spin polarization transverse to the scattering plane. Scattering of these polarized electrons from the second target results in a leftright scattering asymmetry, again due to the spin-orbit interaction, that is proportional to the polarization induced by the first scattering. Mott's proposal stimulated numerous experimental searches for such an asymmetry, ${ }^{7,8}$ but it was not until 1942 that Schull, et al. ${ }^{9}$ after careful correction for instrumental effects, demonstrated the existence of a scattering asymmetry that was in agreement with Mott's calculated value. The emphasis in Mott scattering studies then shifted from confirmation of fundamental theory to the production and/or measurement of electron polarization in connection with other topics in physics. An early example of this was the double-scattering $g$-factor experiment of Louisell et al. ${ }^{10}$ in which polarized electrons produced by scattering from a foil target were acted on by a magnetic field. Precession of the spin magnetic moment in this field was detected through Mott scattering at a second foil, allowing a determination of the electron's $g$ factor. Following the discovery of parity violation by Wu et al., ${ }^{11}$ it was pointed out that parity violation would require that electrons produced through $\beta$ decay of unaligned nuclei be polarized. ${ }^{12}$ This prompted extensive $\beta$-ray polarization measurements involving, for the first time, the use of Mott scattering solely for the purpose of analysis (as opposed to production) of electron polarization. ${ }^{13-23}$ Indeed, the currently accepted two-component neutrino theory is founded in large part on accurate Mott electron polarimetry. ${ }^{23}$

Today polarimeters based on Moll scattering (or, more simply, Mott polarimeters) are extensively used in atomic and molecular, solid state, nuclear, and high-energy physics. This use results from the increasing realization that much additional information concerning many physical processes can be obtained through spin-dependent measurements, and, importantly, from the development of relatively simple polarized electron sources for use in such studies. ${ }^{24}$ Current applications of Mott polarimeters include the study of spin-dependent effects in atomic collisions, ${ }^{25,26}$ analysis of surface magnetization of solids, ${ }^{27-29}$ investigation of parity violation in high-cnergy nuclear scattering, ${ }^{30}$ precision measurements of the $Z^{\circ}$ mass, ${ }^{31}$ and tests of special relativity. ${ }^{32}$

In this article, we review the basic physics and application of Mott electron polarimetry, pointing out the po- 


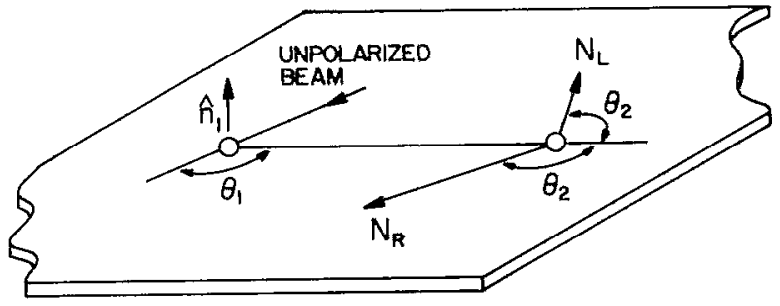

FIG. 1. Schematic diagram of a double-scattering experiment

tential sources of systematic error that can arise in measurements of this type. Several different instrument designs are described that are relatively simple to construct and operate and that provide good efficiency and sizeable scattering asymmetries. The aim is to provide a comprehensive, practical guide to Mott polarimetry and the capabilities of the technique. Although no reviews devoted exclusively to Mott polarimetry have appeared in the literature, the general field of polarized electrons has been reviewed extensively, first by Tolhoek $^{7}$ in 1956. More recent reviews are the two by Farago, ${ }^{33,34}$ and that of Kessler, ${ }^{8}$ which deals with low-energy Mott scattering. The monograph of Kessler ${ }^{25}$ provides the most comprehensive introduction to the physics of spin-polarized free electrons; Chapters 3 and 8 contain discussions of Mott scattering and polarimetry. Other works which review Mott polarization experiments, primarily with respect to $\beta$-ray measurements, are those of Frauenfelder and Rossi, ${ }^{35}$ Frauenfelder and Steffan, ${ }^{36}$ and Schopper. ${ }^{37}$ In addition, a number of journal articles provide particularly useful overviews of the field of Mott polarimetry, insights into specific problems, and/or detailed scientific discussions of important problems. ${ }^{17.18,38-48}$

\section{PHYSICAL PRINCIPLES}

The physical basis of Mott scattering is perhaps best understood using a classical picture. Consider initially the scattering of a high-energy electron by a bare nucleus of charge $\mathrm{Ze}$. (This represents a reasonable model for largeangle scattering of high-energy electrons by high- $Z$, atoms, because for such scattering to occur an electron must be incident with a small impact parameter.) The motion of the electron in the electric field $\mathbf{E}$ of the nucleus results in a magnetic field $\mathbf{B}$ in the electron rest frame given by

$$
\mathbf{B}=-\frac{1}{c} \mathbf{v} \times \mathbf{E},
$$

where $\mathbf{v}$ is the electron velocity. If $\mathbf{r}$ is the nucleus-electron separation, $\mathbf{E}=\left(Z e / r^{3}\right) \mathbf{r}$ and $\mathbf{B}$ may be written as

$$
\mathbf{B}=\frac{Z e}{c r^{3}} \mathbf{r} \times \mathbf{v}=\frac{Z e}{m c r^{3}} \mathbf{L}
$$

where $\mathbf{L}=m \mathbf{r} \times \mathbf{v}$ is the electron orbital angular momentum. The interaction of this magnetic field with the electron (spin) magnetic moment $\mu_{s}$ introduces a term $V_{\text {so }}=-\mu_{s} \cdot \mathbf{B}$ in the scattering potential. The electron magnetic moment is related to the electron spin $\mathbf{S}$ by $\boldsymbol{\mu}_{s}$ $=-(g e / 2 m c) \mathbf{S}$, where $g$ is the spin $g$ factor $(g \sim 2)$. $V_{\text {so }}$ is therefore given by

$$
V_{\mathrm{so}}=\frac{\mathrm{Ze}^{2}}{2 m^{2} c^{2} r^{3}} \mathbf{L} \cdot \mathbf{S}
$$

where an additional factor of $1 / 2$ has been included to take into account Thomas precession. ${ }^{49}$ The presence of the spin-orbit term $V_{\mathrm{so}}$ in the scattering potential introduces a spin dependence in the scattering cross section $\sigma(\theta)$ which may be written 25

$$
\sigma(\theta)=I(\theta)[1+S(\theta) \mathbf{P} \cdot \hat{n}],
$$

where $S(\theta)$ is the asymmetry function, $I(\theta)$ the spin-averaged scattered intensity, and $\mathbf{P}$ the incident electron polarization. The unit vector $\hat{n}$ is normal to the scattering plane and is defined through the relation

$$
\hat{n} \equiv \frac{\mathbf{k} \times \mathbf{k}^{\prime}}{\left|\mathbf{k} \times \mathbf{k}^{\prime}\right|}
$$

where $\mathbf{k}$ and $\mathbf{k}^{\prime}$ are the wave vectors associated with the incident and scattered electrons, respectively. The direction of $\hat{n}$, which is parallel to $\mathbf{L}$, depends on whether scattering to the left or right is being considered.

Consider again the double-scattering experiment shown in Fig. 1. Because the first scattering is to the left, the normal $\hat{n}_{1}$ to the scattering plane will be directed as shown in the figure. The unpolarized incident electron beam can be considered as comprising equal numbers of electrons with spins parallel and antiparallel to $\hat{n}_{1}$, i.e., with spin-up $\left(m_{s}=+1 / 2\right)$ and spin-down $\left(m_{s}=\right.$ $-1 / 2$ ). From Eq. (4) it follows that the number $N_{\uparrow}$ of spin-up electrons scattered (to the left) through angle $\theta_{1}$ is proportional to $1+S\left(\theta_{1}\right)$, whereas the number $N_{1}$ of scattered spin-down electrons is proportional to 1 - $S\left(\theta_{1}\right)$.Thus electrons scattered through $\theta_{1}$ have a net polarization $P\left(\theta_{1}\right)$ given by

$$
P\left(\theta_{1}\right)=\frac{N_{\uparrow}-N_{\uparrow}}{N_{\uparrow}+N_{\uparrow}}=S\left(\theta_{1}\right),
$$

or, in vector notation, $\mathbf{P}\left(\theta_{1}\right)=S\left(\theta_{1}\right) \hat{n}_{1}$. Scattering of these polarized electrons from a second target results in a left-right scattering asymmetry $A\left(\theta_{2}\right)$ defined as

$$
A\left(\theta_{2}\right)=\frac{N_{L}-N_{R}}{N_{L}+N_{R}}
$$

where $N_{L}$ and $N_{R}$ are the number of electrons scattered to the left and right, respectively, through angle $\theta_{2}$. If the first and second scattering events are coplanar, $N_{L}$ will be proportional to $N_{1}\left[1+S\left(\theta_{2}\right)\right]+N_{1}\left[1-S\left(\theta_{2}\right)\right]$, whereas $N_{R}$ will be proportional to $N_{t}\left[1-S\left(\theta_{2}\right)\right]+N_{\mathrm{t}}[1$ $+S\left(\theta_{2}\right)$ ]. Substitution in Eq. (7) yields

$$
A\left(\theta_{2}\right)=P\left(\theta_{1}\right) S\left(\theta_{2}\right) \text {. }
$$

This relation forms the basis of Mott electron polarimetry. If the asymmetry function $S\left(\theta_{2}\right)$ is known, measurement of the scattering asymmetry $A\left(\theta_{2}\right)$ yields $P\left(\theta_{1}\right)$, i.e., the component of incident beam polarization perpendicular to the scattering plane. 


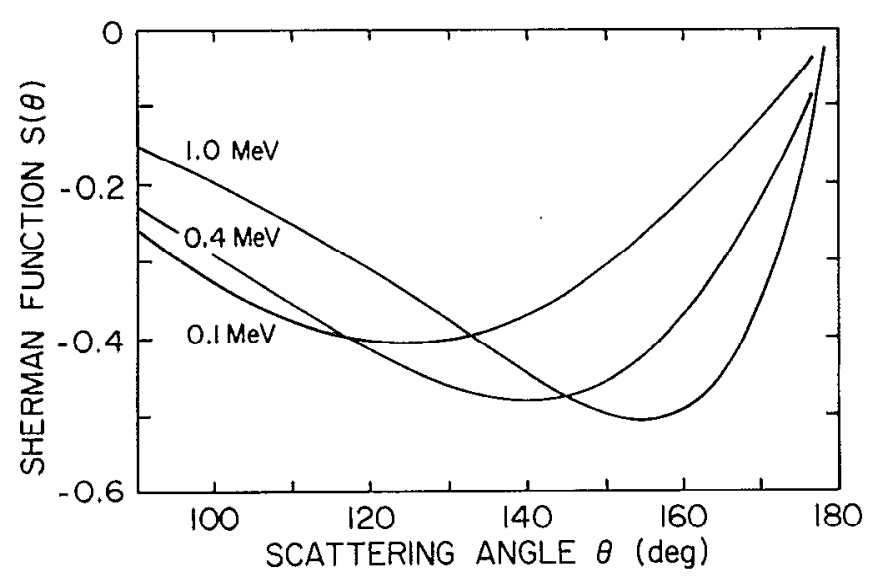

FIG. 2. Angular and energy dependence of the Sherman function for scattering from gold (taken from Ref. 51).

Calculations of asymmetry functions $S(\theta)$ for highenergy electron scattering have been extensive, starting with the initial work of Mott. ${ }^{4,5}$ Sherman undertook comprehensive calculations of relativistic electron scattering from a point Coulomb field and for this reason $S(\theta)$ is frequently termed the Sherman function. ${ }^{50}$ The general characteristics of $S(\theta)$ for scattering from gold nuclei are illustrated in Fig. $2 .{ }^{51}$ [As suggested by Eq. (3), the spinorbit effect is largest for high- $Z$ targets]. For large scattering angles, $S(\theta)$ can be sizable implying that under appropriate conditions the left-right asymmetry in the scattering of polarized electrons should be readily detectable. Subsequent calculations of $S(\theta)$ have considered scattering from atoms and include effects due to inner shell screening. ${ }^{52-54}$

Although spin-dependent effects in scattering from nuclei become negligible at low ( $\lesssim 1 \mathrm{keV}$ ) energies, asymmetries in low-energy scattering from atoms can still be appreciable, as was first suggested by calculations of Massey and Mohr. ${ }^{55}$ This results because at low energies the deBroglie wavelength of the electrons becomes comparable to atomic dimensions and the scattering process can be considered as a diffractive one. As such, the differential scattering cross section displays oscillatory behavior with scattering angle $\theta$ and is spin sensitive due to the presence of $V_{\mathrm{so}}$ in the scattering Hamiltonian. This is illustrated in Fig. 3 which shows calculated elastic differential scattering cross sections for spin-up and spin-down electrons incident at $300 \mathrm{eV}$ on mercury atoms. ${ }^{8}$ For certain values of $\theta$ these cross sections are significantly different, resulting in large Sherman function values $S(\theta)$ that, as shown in Fig. 3, can approach unity. Unfortunately the maxima in $S(\theta)$ occur near local minima in the differential scattering cross section where the scattering efficiency is low. Calculations of $S(\theta)$ for low-energy electron scattering by a variety of atoms including $\mathrm{Hg}, \mathrm{Pb}$, and $\mathrm{Xe}$ have been reported. ${ }^{8,56-59}$

The Sherman function must be measured using either a double-scattering experiment, or by using a beam of electrons of known polarization and observing the scattering asymmetry. Double-scattering experiments performed to date have used as targets either $\mathrm{Hg}$ beams ${ }^{60}$ or $\mathrm{Au}$ films. ${ }^{38,45-47,61-66}$ In general, measurements using atomic

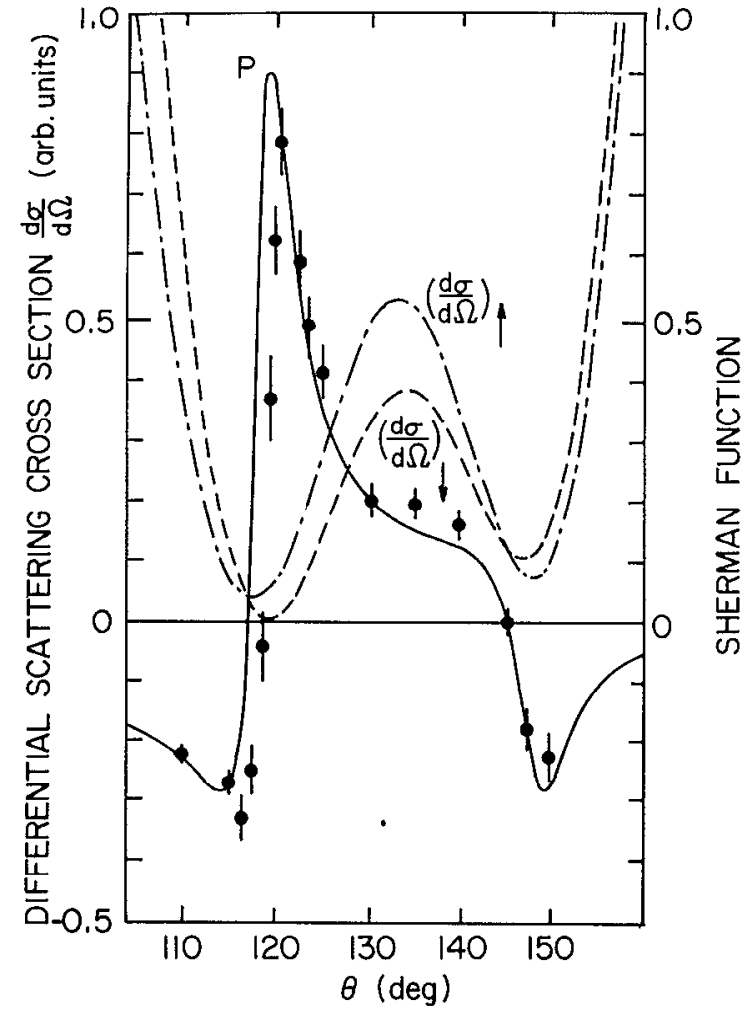

FIG. 3. (a) Calculated elastic differential scattering cross sections ( $d \sigma /$ $d \Omega$ ) for spin up and spin down electrons incident at $300 \mathrm{eV}$ on mercury atoms. (b) Sherman function $S(\theta)$; - theory; $\theta$, experimentally measured polarization $P(\theta)$ of electrons elastically scattered from mercury atoms $[P(\theta)=S(\theta)$; see Eq. (6)]. The data are taken from Ref. 8 .

beams yield values of $S(\theta)$ in good agreement with theory for electron energies $\gtrsim 100 \mathrm{eV}$. In the case of high-energy scattering from foil targets effects due to plural scattering (defined generally to be a small number of large-angle scatterings) and multiple scattering (defined to be a large number of small-angle scatterings) within the foil must be considered and tend to reduce the measured "effective" Sherman functions $S_{\text {eff }}(\theta)$ below those calculated for single atom scattering. This problem, and the accuracy of the theoretical calculations, will be considered in detail later. A number of measurements of $S_{\text {eff }}(\theta)$ for Au films using incident electrons of known polarization have also been reported. $19,67-70$

An important parameter in discussing the performance of Mott polarimeters is the efficiency $\epsilon$, defined $a^{25}$

$$
\epsilon=\left(\frac{I}{I_{0}}\right) S_{\text {eff }}^{2}
$$

where $I_{0}$ is the current entering the polarimeter, $I$ is the total scattered current measured by the left and right detectors, and $S_{\text {eff }}$ is the analyzing power of the apparatus. The quantity $\epsilon$, also referred to as the "figure of merit," is proportional to the inverse square of the statistical error in an electron counting experiment to measure the polarization $P$ of an incident beam. Thus maximization of $\epsilon$ minimizes the error in the measured value of $P$ for a given number of incident electrons. 


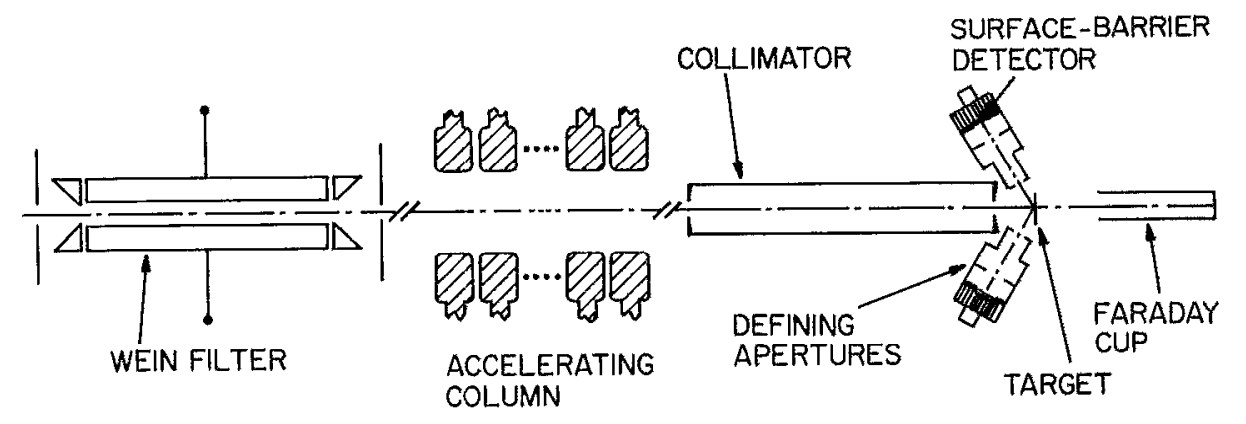

FIG. 4. Schematic diagram of a conventional high-energy Mott polarimeter (see Ref. 71).

\section{MOTT POLARIMETER DESIGNS}

Practical Mott polarimeters have been realized that employ a wide range of geometries and operating energies. Here we describe a representative sample of these that illustrate a number of design and performance considerations. The majority of these instruments involve scattering from gold targets. Gold is selected because it has a high $Z$ $(=79)$ and hence a large Sherman function $S$, because it is nonreactive and does not form a thick oxide layer, and because thin gold films, which reduce multiple and plural scattering, are easy to fabricate. In the case of retardingpotential polarimeters, however, use of uranium and thorium targets offers some advantages. Polarimeters based on scattering from mercury vapor will also be described.

\section{A. "Conventional" Mott polarimeters}

A schematic diagram of a "conventional" high-energy Mott polarimeter used in field emission studies ${ }^{71}$ is presented in Fig. 4, and is representative of those used in a number of laboratories. ${ }^{42,72-94}$ It comprises a spin rotator, an electrostatic accelerating column, and a scattering chamber maintained at high potential. Electrons entering the device first pass through a Wein filter which can be used, for example, to change their polarization from longitudinal to transverse, as required for Mott scattering. The electrons are accelerated to $100 \mathrm{keV}$ by passage through an accelerating column and, after collimation, scatter from a gold target foil. Electron scattering energies of $\sim 100 \mathrm{keV}$ are employed in most conventional Mott polarimeters. Although use of higher electron energies would result in higher Mott scattering asymmetries (see Fig. 2), the gain in the Sherman function is more than offset for energies $\gtrsim 150 \mathrm{keV}$ by increased experimental difficulties and by reductions in the scattering cross section which lead to lower signal rates. Use of electron energies below $\sim 50 \mathrm{keV}$ results in a substantial reduction in $S$ and increased problems with multiple and plural scattering in the target.

To minimize the effects of multiple and plural scattering, very thin gold targets are employed that are produced by evaporating gold onto low-molecular-weight hydrocarbon (e.g., Formvar) carrier foils. The target foils are mounted on a wheel allowing targets of different thickness to be positioned in the beam for purposes of calibration (as will be discussed in the next section). Those electrons that pass through the target foil are collected by a Faraday cup. Electrons scattered through $\sim \pm 120^{\circ}$ (where the Sherman function has a broad maximum) are detected by two symmetrically positioned silicon surface barrier (SSB) detectors and the scattering asymmetry $A$ is measured. This asymmetry gives directly the component of spin polarization perpendicular to the scattering plane via the relation $A=P S_{\text {eff }}$ [see Eq. (8)]. Typical SSB detectors provide energy resolutions of $\sim 10 \mathrm{keV}$ and afford some measure of discrimination against inelastically scattered electrons. In addition, they are insensitive to $\gamma$ rays and provide low background count rates. Often four SSB detectors are employed, located at azimuthal angles of $0^{\circ}, 90^{\circ}, 180^{\circ}$, and $270^{\circ}$, so that both transverse components of the electron polarization can be determined simultaneously. This capability, in conjunction with a Wein filter, permits measurement of the full vector polarization $\mathbf{P}$ of an incident beam. ${ }^{76}$

Variations on the design shown in Fig. 4 have included the use of concentric hemispherical electrodes ${ }^{78}$ to accelerate the electrons and the use of scintillators coupled to photomultiplier tubes to detect the scattered electrons. ${ }^{83}$ In addition, a number of polarimeters have employed detectors placed symmetrically behind the target foil. ${ }^{75,90} \mathrm{Be}$ cause the Mott asymmetry is very small for forward scattering, such detectors can be used to monitor and eliminate instrumental asymmetries. A segmented Faraday detector placed directly behind the target foil has been used for the same purpose. ${ }^{82}$ As an alternate to the use of a Wein filter, spin rotation has also been achieved by electrostatic deflection and by $90^{\circ}$ scattering from bulk graphitic carbon or a gold foil. $\left(90^{\circ}\right.$ Coulomb scattering of longitudinally polatized electrons from atomic nuclei leaves the polarization vector largely unchanged. ${ }^{53,95}$ )

The principal disadvantages of conventional Mott polarimeters are that the target and electron detectors must be operated at high potential and that discrimination against inelastically scattered electrons is poor. They do, however, provide very high efficiencies $\epsilon$. Efforts to maximize $\epsilon$ have involved enhancing $I / I_{0}$, the ratio of scattered to incident beam currents. This can be accomplished by increasing the solid angle subtended by the detectors at the target surface, which is simply achieved by placing the detectors close to the target, or by increasing the thickness of the target. The latter can result in a significant decrease in $S_{\text {eff }}$ due to plural and multiple scattering, and it is necessary to compromise between the increase in $I / I_{0}$ and decrease in $S_{\text {eff }}$. With a careful choice of operating parameters, however, conventional high-voltage Mott polarimeters can provide efficiencies $\epsilon \gtrsim 1 \times 10^{-4}$. 


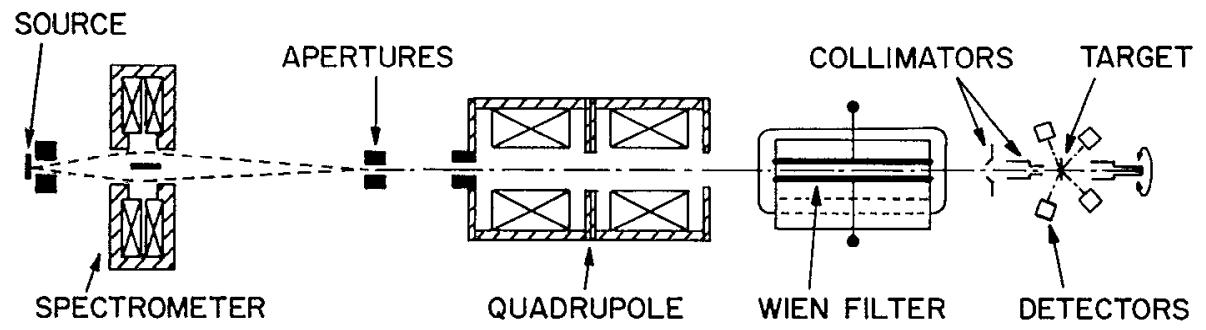

FIG. 5. Schematic diagram of a $\beta$-ray polarimeter. The scattered electron detectors are drawn rotated by $90^{\circ}$ (see Ref. 105).

\section{B. $\beta$-ray polarimeters}

One interesting subset of conventional Mott polarimeter designs are those developed specifically for $\beta$-ray studies. ${ }^{13-19,21,22,96-105}$ Typically, since $\beta$ rays are formed with high energy, no electron acceleration is required permitting some apparatus simplifications (see, for example, Ref. 15). A modern example of a $\beta$-ray polarimeter is shown in Fig. 5. ${ }^{105}$ Beta rays from the source of interest pass through a short lens spectrometer which provides initial velocity selection and increases the solid angle over which source electrons are collected. The electrons are then focused by a quadrupole doublet to guide them through a Wein filter for spin rotation ( $\beta$ rays are longitudinally polarized) and further velocity selection. The electrons are subsequently collimated by a series of apertures and strike the gold target foil. Those scattered by $\sim \pm 120^{\circ}$ are detected by two SSB detectors. Additional SSB detectors are placed at forward scattering angles to aid in monitoring instrumental asymmetries. The entire detector assembly can be rotated by $180^{\circ}$ about a longitudinal axis to interchange the left and right detectors and thus identify instrumental asymmetries associated with different detector solid angles and efficiencies.

As with conventional Mott detectors, $\beta$-ray polarimeters have used both scintillation and SSB detectors, although many early instruments employed Geiger counters. Electrostatic deflection and Coulomb scattering from foils have also been used for spin rotation. Some instruments have included provisions for electrostatic acceleration of the electrons. This is advantagcous bccause it allows Mott scattering to be undertaken at a selected energy where the combination of efficiency, $S_{\text {eff }}$ and the electron optical properties of the apparatus is optimal. Acceleration is particularly important, for example, in the study of $\beta$ rays from ${ }^{3} \mathrm{H}_{;}^{22}$ the end point energy of this decay is $18 \mathrm{keV}$ and acceleration is required to obtain an acceptable $S_{\text {eff. }}$ No estimates of efficiencies for $\beta$-ray polarimeters have been published.

\section{Retarding-potential Mott polarimeters}

In recent years, based on an initial suggestion by Farago, a range of increasingly compact retarding-potential Mott polarimeters have been developed. ${ }^{40,41,70,106-111}$ In these polarimeters the incident electrons are accelerated by an electric field established between an inner and outer electrode. The outer electrode is operated near ground potential, the inner electrode at a large positive potential. At the center of the hollow inner electrode is a gold target and those electrons scattered through $\pm 120^{\circ}$ exit the inner electrode and are decelerated as they pass again to the outer electrode. Scattered electrons with sufficient energy to overcome the retarding field are detected by two symmetrically positioned channeltrons.

A schematic diagram of a typical cylindrical-geometry retarding-potential polarimeter ${ }^{48}$ is shown in Fig. 6. The inner electrode is mounted on a high voltage insulator and is typically operated at $\sim 100 \mathrm{kV}$. Besides relative compactness, retarding potential analyzers have the advantage that they provide excellent discrimination against inelastically scattered electrons and that the scattered electron detectors and other major portions of the apparatus are at ground potential. Further, because of the strong radial field between the inner and outer cylinders, the incident beam is strongly focused resulting in a very stable beam position on the target foil. The inelastic energy loss that an electron can suffer and still be detected, i.e., the inelastic energy loss window $\Delta E$, can be adjusted by varying the bias applied to the channeltron cones and to the apertures placed in front of them. With careful design of the retarding field electrodes energy resolutions $\lesssim 3 \mathrm{eV}$ can be attained. This is illustrated in Fig. 7, which shows the energy distribution of electrons scattered at $100 \mathrm{keV}$ from a thin gold target. The elastic scattering peak is clearly resolved.

Another particular advantage of high-voltage cylindrical polarimeters is that, if a very thin target foil is used, the majority of the incident electrons will pass through the target foil without significant scattering and can transit the entire instrument with little degradation in beam quality or energy distribution. Thus devices of this type can be used "in line," allowing the beam polarization to be measured at the same time that the analyzed beam is being used in some other experiment.

Operation at high energies has the advantage that the corresponding values of $S_{\text {eff }}$ are relatively large, while the effects of plural and multiple scattering in the target are minimized, which makes high precision polarization measurements easier. However, it also leads to low efficiencies because the scattering cross section decreases with increasing energy and because electrical breakdown considerations dictate a relatively large spacing between the inner and outer cylinders which reduces the solid angle subtended by the electron detectors. To improve the efficiency and compactness of retarding-potential polarimeters, instruments operating at lower voltages have been developed based on both spherical and conical geometries. Two such instruments are shown in Figs. 8 and 9 and use accelerating voltages of 40 and $20 \mathrm{kV}$, respectively.

The spherical design ${ }^{107}$ provides two-dimensional focusing of the incident beam resulting in a very well-defined 


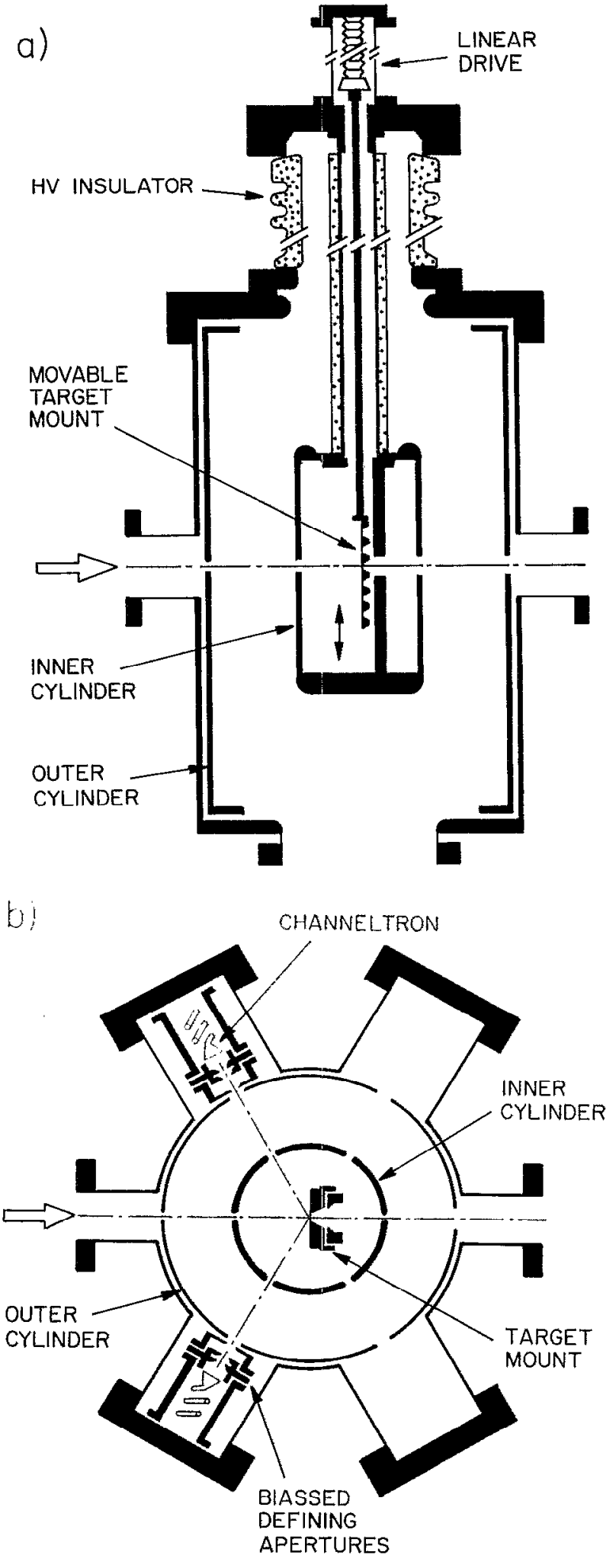

FIG. 6. (a) and (b) Schematic diagram of a cylindrical retarding potential polarimeter (sec Ref. 48).

impact point on the target. In the smaller conical polarimeter ${ }^{108}$ the incident beam is focused on the target by a three-element coaxial electrostatic lens whose final element is at the scattering potential. The retarding field is

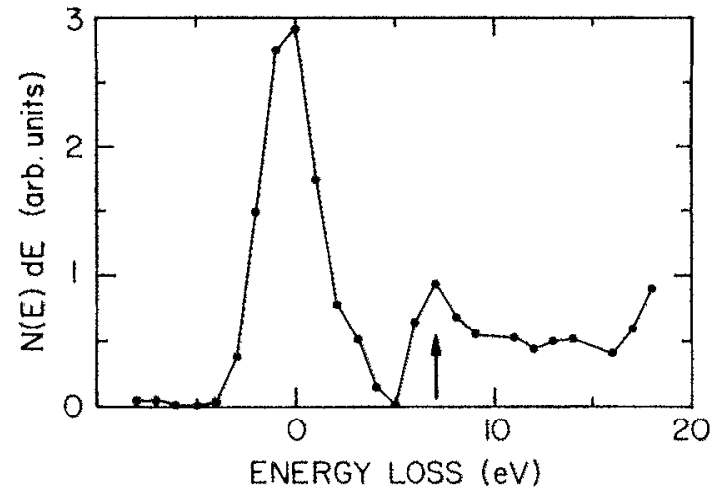

FIG. 7. Measured energy distribution of electrons scattered at $100 \mathrm{keV}$ from a thin gold target (Ref. 48). The arrow indicates a surface plasmon feature.

established using planar electrodes. Each geometry allows the use of four scattered electron detectors permitting simultaneous measurement of both transverse components of beam polarization. The good energy resolution inherent with these designs also permits the use of bulk targets without significant loss of performance. ${ }^{112}$ Conical polarimeters can be made sufficiently small that they are readily moved within a vacuum system, making possible angle-resolved polarization measurements. ${ }^{113,114}$ An interesting design variation on the spherical configuration has been developed which employs a spherical inner and a cylindrical outer electrode. ${ }^{70}$

The efficiencies of retarding-potential polarimeters tend to be low. The ratio $I / I_{0}$ of the scattered and incident

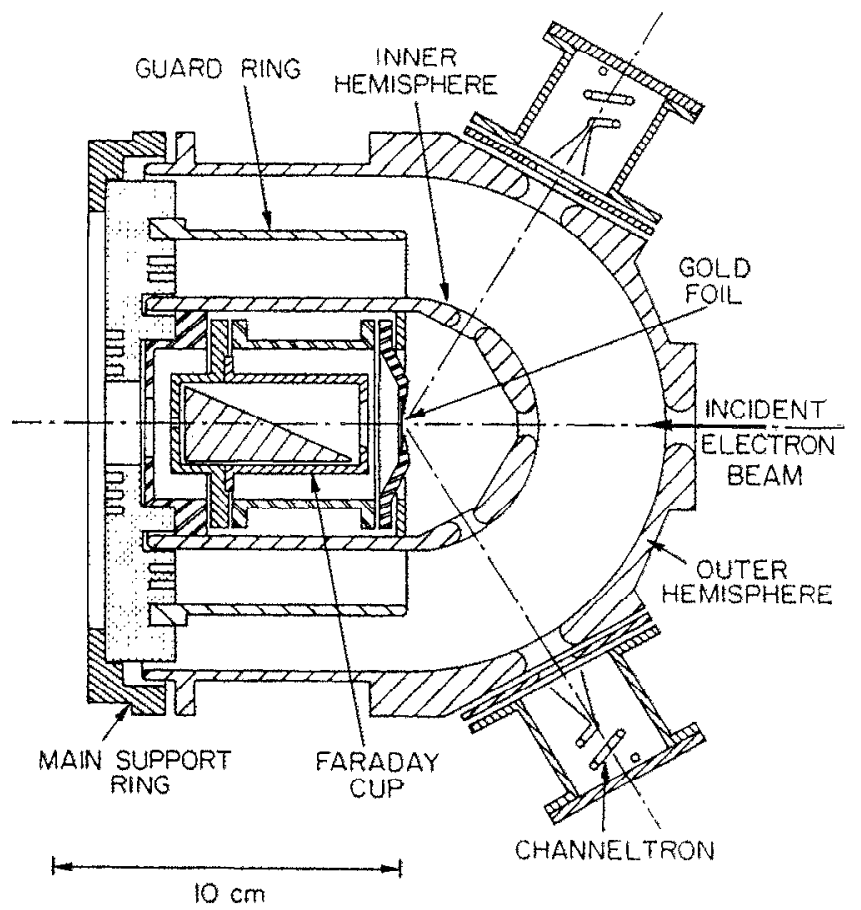

FIG. 8. Schematic diagram of a spherical retarding potential polarimeter (taken from Ref. 107). The major components of the apparatus are symmetrical about the horizontal axis. 


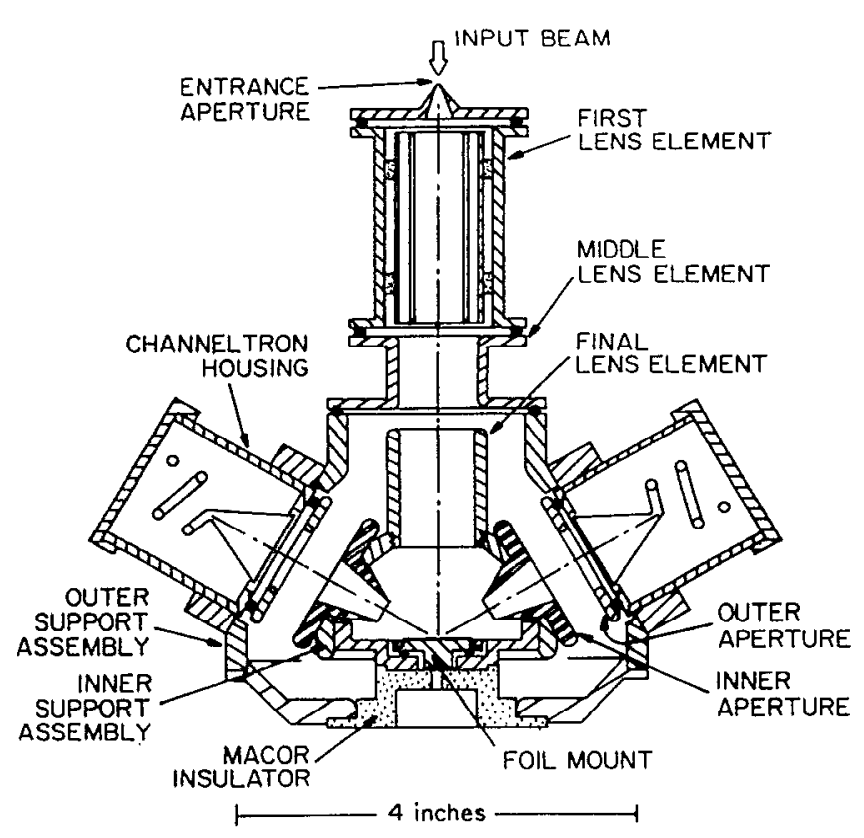

FIG. 9. Schematic diagram of a compact retarding potential polarimeter. The major components of the apparatus are symmetrical about the vertical axis (taken from Ref. 108).

currents can be increased by increasing the energy loss window $\Delta E$ (typically values of $\Delta E$ in the range $0.5-1.3$ $\mathrm{keV}$ are used) and/or by lowering the scattering energy, although the resultant improvements in $I / I_{0}$ are gained at the expense of a decrease in $S_{\text {eff }}$. Cylindrical analyzers operating at $\sim 100 \mathrm{keV}$ provide efficiencies of $\sim 10^{-7}$ but values approaching $\sim 3 \times 10^{-5}$ have been achieved ${ }^{108}$ with compact polarimeters operating at $20 \mathrm{keV}$. These efficiencies can be further improved by use of higher- $Z$ target materials, specifically thorium $(Z=90)$ and uranium ( $Z$ =92). This results in an increase in both $S_{\text {eff }}$ and $I / I_{0}$ because the spin-orbit effect and differential scattering cross sections each increase with $Z$. Use of a bulk thorium target ${ }^{69,106}$ increases $S_{\text {eff }}$ by $\sim 20-30 \%$ and $I / I_{0}$ by $\sim 15 \%$ relative to gold, resulting in nearly a factor 2 gain in efficiency. A similar improvement can be realized using a uranium target. ${ }^{110}$ Although thorium and uranium are more reactive than gold, stable values of $S_{\text {eff }}$ can be obtained following careful cleaning and passivation of the target surface.

\section{Low-energy diffuse scattering Mott polarimeter}

Mott polarimeters based on low-energy $(150 \mathrm{eV})$ diffuse scattering from an amorphous gold surface have been developed that combine high efficiency with small size. ${ }^{44,115-117} \mathrm{~A}$ recent design is shown schematically in Fig. $10 .{ }^{116}$ Incident electrons are accelerated and focused onto an evaporated polycrystalline gold target by the input optics. The target, drift tube, and electrode $E_{2}$ coplanar with the target are all maintained at the same potential so that scattering occurs in a nearly field-free region. A negative bias applied to the focusing electrode $E_{1}$, and a positive bias applied to the grid $G_{1}$, are used to deflect the scattered electrons such that, upon arrival at $G_{1}$, their trajectories

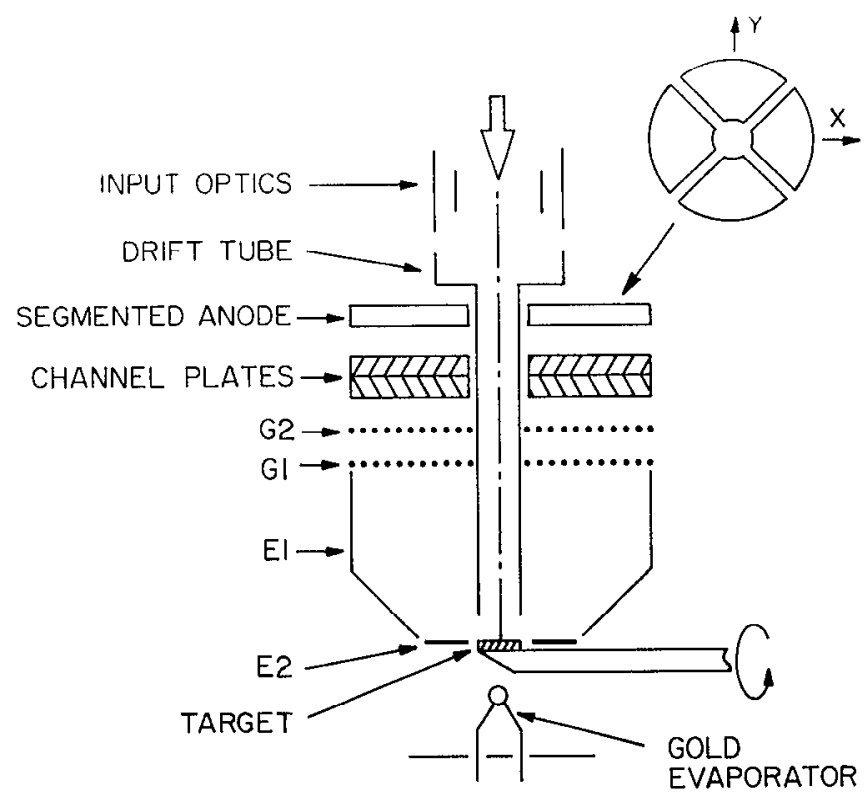

FIG. 10. Cross section of a low-energy diffuse scattering polarimeter. The major components of the apparatus are symmetrical about the vertical axis (taken from Ref. 116).

are approximately perpendicular to $G_{1}$. A negative bias is applied to $G_{2}$ to discriminate against incident electrons that suffer large inelastic energy losses upon scattering and true secondary electrons produced by incident electron impact on the target. Those electrons that are able to pass through $G_{2}$ are accelerated and detected by an annular dual-chevron microchannel plate with a four quadrant anode. As indicated in the inset in Fig. 10, each quadrant subtends an azimuthal angle of $90^{\circ}$ permitting simultaneous measurement of both transverse components of incident beam polarization.

Low-energy diffuse scattering Mott polarimeters provide very good efficiencies. This results because the design allows collection of electrons scattered over a wide range of angles. The performance is further enhanced by carefully selecting the cut-off energy for the scattered electrons by ensuring that their trajectories are close to perpendicular to the grids. Selection of the minimum and maximum scattering angles subtended by the grids is fixed by the entrance drift tube and $E_{1}$. Under optimum operating conditions efficiencies $\epsilon$ of $\sim 2 \times 10^{-4}$ have been achieved. Low-energy electron scattering is, however, influenced by the presence of adsorbed layers on the target surface, which must therefore be periodically renewed. The scattering is also strongly influenced by multiple and plural scattering which, in conjunction with the broad spatial and energy profiles of the scattered electrons, effectively eliminates any possibility of self calibration of the device.

\section{E. Mercury-vapor Mott polarimeters}

A number of low-energy polarimeters based on scattering from a mercury atom beam or vapor have been described. ${ }^{60,118-125}$ Because of their low voltage operation, such polarimeters can be made quite compact, within the 


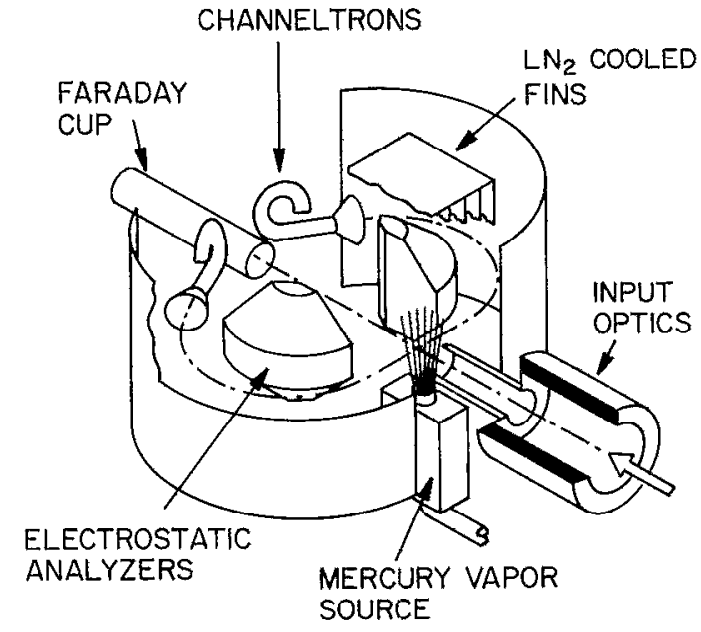

FIG. 11. Schematic diagram of a mercury vapor polarimeter (see Ref. 125).

mechanical limits imposed by the mercury target sources themselves. The complexity of these sources represents the major disadvantage of this class of analyzers.

A particularly efficient mercury-vapor polarimeter is shown schematically in Fig. $11 .^{125}$ The incident electron beam is scattered from a mercury atom beam produced by upward effusion from a vertical nozzle that is supplied by a reservoir. The atom beam is condensed on a set of liquid$\mathrm{N}_{2}$-cooled fins. Electrons scattered at $\sim \pm 90^{\circ}$ enter two symmetrically positioned quasispherical electrostatic analyzers and are focused onto channeltrons. These analyzers are specifically designed to increase the solid angle over which scattered elcctrons are detceted, and thus increase efficiency. They also shield the channeltrons from UV photons created by electron impact excitation of the mercury target and provide some discrimination against inelastically scattered electrons. Incident electrons that traverse the target beam are collected in a Faraday cup.

The performance of the instrument was evaluated at scattering energies of 15 and $300 \mathrm{eV}$. Because of uncertainties in the electron angular acceptance characteristics (electrons scattered through angles of $\sim 85^{\circ}-100^{\circ}$ are collected), it is not possible to calculate accurate effective Sherman functions from atomic scattering data. The values of $S_{\text {eff }}$ were therefore measured directly using a doublescattering approach yielding $S_{\text {eff }}=0.37$ and -0.17 at 15 and $300 \mathrm{eV}$, respectively. The corresponding ratios $I / I_{0}$, which depend on the target density, were $2.8 \times 10^{-4}$ and $6.7 \times 10^{-5}$. The resulting efficiencies of $\sim 4 \times 10^{-5}$ at 15 $\mathrm{eV}$ and $\sim 2 \times 10^{-6}$ at $300 \mathrm{eV}$ are comparable to those of retarding-potential polarimeters. The maximum target density, however, was limited by vacuum problems associated with the design of the nozzle suggesting that higher efficiencies might be realized by use of an improved target beam source.

In other mercury vapor polarimeter designs ${ }^{122}$ defining apertures are used to ensure that only those electrons elastically scattered at a well defined angle are detected. This reduces $I / I_{0}$, and thus the efficiency, but can remove the need for calibration, i.e., $S_{\text {eff }}$ may be taken as equal to the corresponding atomic Sherman function $S$.

\section{OPERATION}

In this section we examine sources of systematic error that can affect the accuracy and precision of Mott polarization measurements. Since such measurements require determination of a left-right scattering symmetry $A$ and knowledge of the effective Sherman function $S_{\text {eff }}$ uncertainties in both will contribute to the overall uncertainty in the measured polarization. Even for an unpolarized incident electron beam the measured scattering asymmetry may be nonzero due to instrumental asymmetries associated with beam or apparatus misalignment, beam and/or target inhomogeneities, unequal detector responses, stray fields, etc. Clearly such instrumental asymmetries must be identified and taken into account. Spurious background signals at the detectors can also contribute to the uncertainty in $A$. Errors in $S_{\text {eff }}$ can result both from experimental uncertainties associated with the particular calibration procedure employed and, if the calibration involves normalization to theoretical values, from uncertainties associated with the theory.

\section{A. Measurement of scattering asymmetries}

\section{Instrumental asymmetries}

Instrumental asymmetries in conventional high-energy Mott polarimeters have been considered in detail elsewhere ${ }^{17,18,25,38,46,47}$ and only a brief overview is presented here. Such asymmetries can result from unequal detector responses and/or apparatus misalignment and will be discussed by reference to Fig. 12. The ideal experimental geometry is shown in Fig. 12(a). The input beam is incident along the system axis and the symmetrically positioned detectors define equal scattering angles $\theta$ and subtend equal solid angles at the target a distance $d$ away. As illustrated in Fig. 12(b), misalignment may result in the incident beam being inclined at some angle $\phi$ to the instrumental axis and striking the target a distance $\Delta s$ from its center. The solid angle subtended by the left detector is increased by an amount

$$
\frac{\Delta \Omega}{\Omega}=\frac{-2 \Delta d}{d}=\frac{2 \Delta s}{d} \sin \theta,
$$

and the scattering angle $\theta$ to the left detector is increased by

$$
\Delta \theta=\phi-\frac{\Delta s}{d} \cos \theta
$$

Analogous changes of opposite sign occur for the right detector. (Similar effects can also be introduced by beam or target inhomogeneities.) Thus with an unpolarized incident beam misalignment will give rise to a false asymmetry which, to first order in $\phi$ and $\Delta s$, may be written ${ }^{46}$

$$
A_{f}=\frac{\Delta \Omega}{\Omega}+\frac{1}{I} \frac{\partial I}{\partial \theta} \Delta \theta
$$



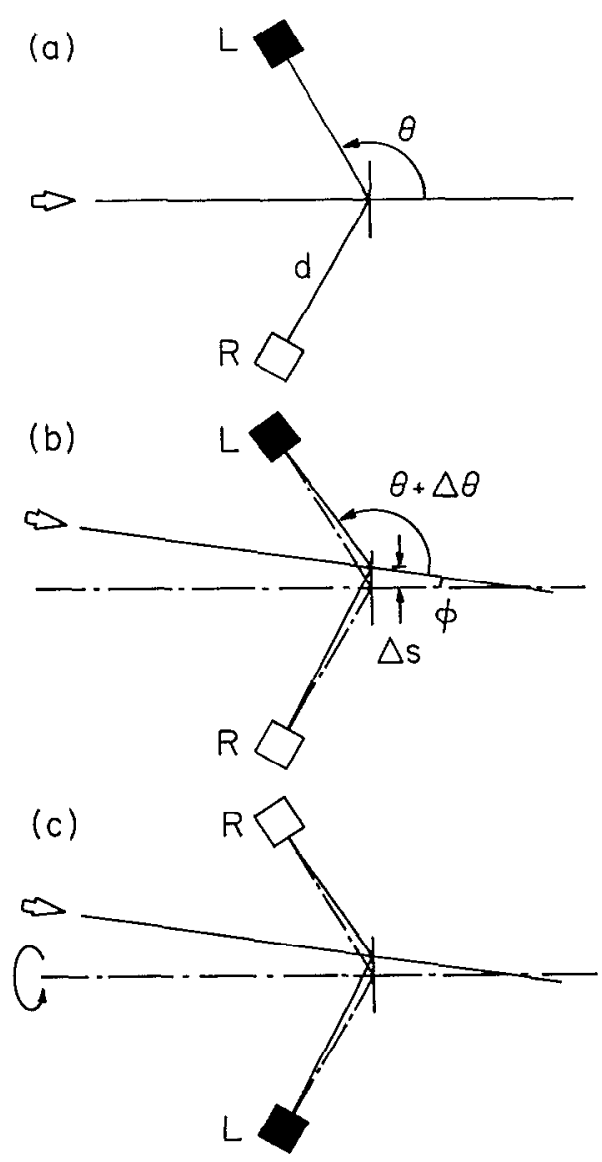

FIG. 12. Instrumental asymmetries in a conventional high-energy Mott polarimeter. (a) Ideal experimental geometry, (b) misaligned input beam, (c) effect of $180^{\circ}$ rotation about polarimeter axis.

$$
=\frac{2 \Delta s}{d} \sin \theta+\frac{1}{I} \frac{\partial I}{\partial \theta}\left(\phi-\frac{\Delta s}{d} \cos \theta\right),
$$

where $(1 / I)(\partial I / \partial \theta)$ is the relative rate of change of the scattered intensity with scattering angle. Because the scattered intensity decreases with increasing scattering angle, this quantity is negative and the two terms present in Eq. (12) tend to cancel.

A number of techniques have been used either to measure instrumental asymmetries or to eliminate their effects. Such elimination is particularly simple in cases where the input beam polarization can be reversed $(P \rightarrow-P)$ without changing the beam trajectory or characteristics. Prior to reversal, the ratio of the counting rates in the left and right detectors is given by

$$
\frac{R_{L}}{R_{R}}=\frac{\eta_{L}}{\eta_{R}} \frac{\left(1+P S_{\mathrm{eff}}\right)}{\left(1-P S_{\mathrm{eff}}\right)} \frac{\left(1+A_{f}\right)}{\left(1-A_{f}\right)},
$$

where $\eta_{L}$ and $\eta_{R}$ represent the detector efficiencies.

If the input beam polarization is reversed the new ratio of the counting rates is

$$
\frac{R_{L}^{\prime}}{R_{R}^{\prime}}=\frac{\eta_{L}}{\eta_{R}} \frac{\left(1-P S_{\mathrm{eff}}\right)}{\left(1+P S_{\mathrm{eff}}\right)} \frac{\left(1+A_{f}\right)}{\left(1-A_{f}\right)} .
$$

Combining these equations it is possible to eliminate the detector efficiencies and false asymmetry and $P$ may be determined directly from

$$
P S_{\mathrm{eff}}=\frac{X-1}{X+1},
$$

where $X \equiv\left(R_{L} R_{R}^{\prime} / R_{R} R_{L}^{\prime}\right)^{1 / 2}$. This technique is particularly simple to apply using polarized electron sources that make use of circularly polarized radiation, such as the GaAs source, because the electron polarization can be accurately reversed simply by reversing the helicity of the radiation. Use of a Wein filter for polarization reversal is more problematic as this might introduce changes in beam trajectory that would also change the instrumental asymmetry.

Unfortunately, in many cases the incoming polarization cannot be reversed and alternate procedures must be used to eliminate instrumental asymmetries. One such procedure is to rotate the detectors through $180^{\circ}$ about the polarimeter axis, as has been frequently done in $\beta$-ray studies. For this procedure to be equivalent to a simple spin reversal the input beam must be incident along the polarimeter axis, which is difficult to ensure. Thus the situation following rotation will typically be as illustrated in Fig. 12(c). The new ratio of the counting rates in the two detectors will be given by

$$
\frac{R_{L}^{\prime \prime}}{R_{R}^{\prime \prime}}=\frac{\eta_{L}}{\eta_{R}} \frac{\left(1-P S_{\mathrm{eff}}\right)}{\left(1+P S_{\mathrm{eff}}\right)} \frac{\left(1-A_{f}\right)}{\left(1+A_{f}\right)} .
$$

It is possible by combining Eqs. (13) and (16) to eliminate the different detector efficiencies but not $A_{f}$, the final result being

$$
P S_{\mathrm{eff}}=\frac{X^{\prime}-1}{X^{\prime}+1} \cdots A_{f}
$$

where $X^{\prime}=\left(R_{L} R_{R}^{\prime \prime} / R_{R} R_{L}^{\prime \prime}\right)^{1 / 2}$ and terms $A_{f} P S_{\text {erf }} \ll 1$ are neglected. In a typical experiment the product $P S_{\text {eff }}$ has magnitude $\sim 0.1, \quad$ corresponding to $P \sim 0.3$ and $S_{\text {eff }} \sim-0.3$. Thus a false asymmetry of only $\sim 0.001$, which corresponds to a very small angular or positional displacement, is sufficient to introduce a fractional error of $\sim 1 \%$ in the measured polarization. Given the difficulty in obtaining precise beam alignment, it is therefore not possible to obtain high accuracy polarization measurements simply by interchanging the left and right detectors.

A number of techniques have been used to measure instrumental asymmetries directly. Perhaps the simplest approach is to replace the target with a low- $Z$ scatterer, such as aluminum, for which $S_{\text {eff }} \sim 0$. Thus any observed asymmetry must be instrumental in origin. ${ }^{14,16,38,40,80}$ Low$Z$ target foils, however, typically have higher transmission coefficients than do those of high- $Z$, which can result in changes in the spurious background signals at the detectors, meaning that the measured asymmetry may not exactly correspond to that appropriate for the high- $Z$ target. An alternate technique that has been adopted to evaluate instrumental asymmetries is to substitute an unpolarized incident electron beam obtained, for example, using a heated filament or a low-energy $\beta$ emitter for which $v /$ 


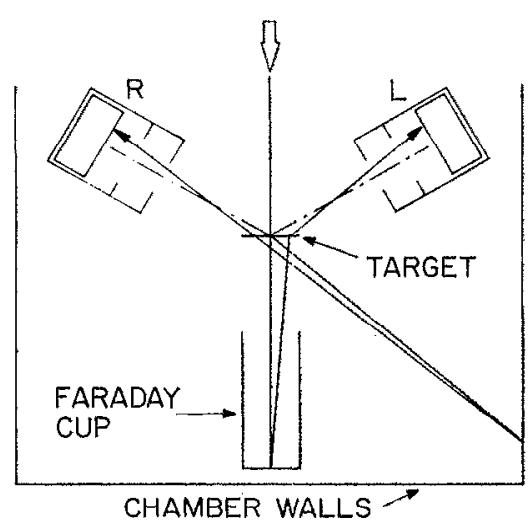

FIG. 13. Electron trajectories that can result in spurious background signals.

$c \sim 0$. This method will, however, only yield the correct asymmetry if the polarized and unpolarized electron beams exactly coincide and have the same spatial characteristics, and this is difficult to achieve.

False asymmetries can also be removed by use of additional monitor detectors placed symmetrically at small scattering angles, where $S_{\text {eff }}$ is again very small. With proper positioning of these detectors, it is possible to eliminate the false asymmetries measured by the left and right detectors from a measurement of the false asymmetries in the monitor counters. ${ }^{38,46,47}$ Using this technique, the fractional error in measured polarizations due to instrumental asymmetries can be reduced to $\$ 0.3 \%{ }^{46,47}$

To date, no careful analyses of instrumental asymmetries in retarding-potential Mott polarimeters have been reported. Since channeltrons are used as detectors, spindependent detection efficiency is a potential problem but, in the one instance where this has been investigated, no spin dependence was found. ${ }^{126}$ Interesting difficulties are encountered in handling instrumental asymmetries when the polarimeter must have a large electron optical acceptance, such as in scanning electron microscopy with polarization analysis. This issue has been discussed thoroughly with regard to a low-energy diffuse scattering polarimeter. ${ }^{115}$

\section{Detector backgrounds}

Measured asymmetries can also be influenced by spurious background signals. ${ }^{25,61,88}$ Two important (and difficult to assess) sources of background signals are incident electrons that reach the detectors by indirect paths, and electrons scattered from the low- $Z$ carrier foil that must be used to support a very thin high- $Z$ target. As illustrated in Fig. 13, incident electrons might reach the detectors by, for example, first passing through the target with only minimal scattering, backscattering in the Faraday cup and then scattering by $\sim 60^{\circ}$ on a second pass through the target. Another possibility is that an electron initially scatters by $\sim 60^{\circ}$ in passing through the target, backscatters from a chamber surface and passes again through the target to a detector. Since, at $\sim 100 \mathrm{keV}$, the cross section for scattering through $60^{\circ}$ is about an order of magnitude greater than that for scattering through $120^{\circ}$, and backscattering coefficients can be appreciable, backgrounds of this type can be significant. Such backgrounds can be reduced by tight collimation of the incident beam and detectors to better define the scattering geometry, but this is not possible if, for example, high efficiency is a requirement. Another approach is to use Faraday cups that contain oblique surfaces or baffles and/or that are constructed of low- $Z$ materials such as beryllium, for which the backscattering coefficient is small. Backscattering from chamber walls can be minimized by use of materials such as aluminum painted with colloidal graphite.

Electrons that reach the detectors by indirect paths typically suffer significant energy loss and can therefore be discriminated against if the detectors have good energy resolution. Indeed, in retarding-potential Mott polarimeters the problem can be effectively eliminated by operating at small inelastic energy loss windows $\Delta E$. If the energy resolution is poor (as in the case of SSB detectors), a number of detailed issues with regard to background subtraction arise that have been discussed in detail elsewhere. ${ }^{42}$

If very thin high- $Z$ targets supported on low $Z$ carrier foils are used, detector signals arising from scattering by the carrier foil must be identified. This is typically accomplished by measuring the detcetor signals using a blank carrier foil. For extremely thin gold targets, carrier foil contributions can approach $50 \%$ of the detected signal. ${ }^{48}$ It should be remembered that both the indirectly scattered background and the carrier foil contribution will, in general, change with target thickness.

Besides the usual dark counts associated with detector and electronic noise, other specific sources of background must be considered. For example, electron impact on the target can result in the production of high-energy photons or sputtered ions. In retarding-potential Mott polarimeters both effects can result in spurious background signals (positive ions are accelerated to the detectors by the retarding field). These can, however, be identified by setting the retarding bias to reject all scattered electrons and measuring any remaining signals. Positive ion ejection from the target can also be suppressed by use of a suitable bias. ${ }^{40,48,107}$

\section{B. Calibration procedures}

\section{Target thickness extrapolations}

The majority of Mott polarimeters make use of solid targets to increase the scattered electron signal. This, however, has the disadvantage that the detected electrons may have suffered scattering from more than one atom in the target. Electrons may, for example, reach the detectors after three $40^{\circ}$ (elastic) scattering events. Such plural scattering can significantly reduce $S_{\text {eff }}$ because, for gold, the Sherman function at $120 \mathrm{keV}$ for $40^{\circ}$ scattering is only -0.014 whereas for $120^{\circ}$ scattering it is -0.4 . Multiple scattering can also reduce $S_{\text {eff }}$ but is generally less important than plural scattering for total scattering angles $\gtrsim 90^{\circ}$. $36,127,128$ Thus, in general, it is not possible to use for $S_{\text {eff }}$ values of the Sherman function calculated for single 
atom scattering. (The target densities employed in mercury vapor polarimeters are sufficiently low that they operate in the single-scattering regime.) Values of $S_{\text {eff }}$ appropriate to a particular target thickness and set of operating conditions can, however, be obtained by measuring the scattering asymmetry using targets of different thickness and extrapolating to zero thickness where, by definition, scattering from single atoms occurs. The data are then normalized in this limit to the calculated Sherman function.

One difficulty with this approach is that the exact functional dependence of the asymmetry $A$ on target thickness $t$ is not known and if an incorrect form is used to extrapolate the data a systematic error in the intercept (and thus instrumental calibration) will result. ${ }^{42,48}$ Ultimately the proper choice of functional form requires a detailed knowledge of the physics of electron scattering in thin films and of the microscopic morphology of the individual targets being used. The general practice is to use targets of suffcient thinness that some linear fit to the data is justified on statistical grounds. This procedure is based on the fact that first order corrections to $A$, coming from double-scattering events, are linear in $t$. Unfortunately there exists no consensus as to which linear fitting parameters are most appropriate. A number of detailed analyses suggest that $1 / A$ vs $t$ should be plotted, ${ }^{17,48,128,129}$ although other work has indicated that $1 / \sqrt{A}$ vs $t$ can yield a linear fit over a broader range of $t .{ }^{18}$ Other approaches that have been used include plotting $\ln A$ vs $t^{42,84}$ and $A$ vs $t^{41,48,70,103}$ An alternate procedure is to consider $A$ as a function of $R\left(=R_{L}+R_{R}\right)$, the combined counting rate in both detectors (which for small target thicknesses is approximately proportional to $t$ ) and extrapolate to zero counting rate using $A$ vs $R^{16,45,48,61}$ or $1 / A$ vs $R .^{42,48}$ (Use of $R$ rather than $t$ eliminates errors associated with measurement of target thickness. ${ }^{130}$ ) Recent experiments suggest that $A$ vs $R$ together with the phenomenological expression $A$ $=a+b e^{-\lambda t}$, where $a, b$, and $\lambda$ are constants, provide the most appropriate fitting forms. ${ }^{48}$ In any case, all these extrapolation forms will be equally valid and will yield statistically identical intercepts if truly linear scaltering conditions prevail, i.e., if higher order plural and multiple scattering are negligible when compared to double scattering. This condition has rarely been met experimentally, as is shown by the failure of most extant data sets in the literature to yield statistically identical intercepts independent of the fitting parameters used. It has been suggested, therefore, that in assigning an error to the intercept this be chosen so as to encompass the results obtained with each fitting procedure. This typically introduces an error of a few percent in the measured values of $S_{\text {eff. }}{ }^{42}$

To obtain very precise extrapolations linear scattering conditions must be established. The relative importance of higher order scattering effects can, in general, be decreased by increasing the electron energy $E,,^{7,14,128,129}$ by reducing the maximum foil thickness used in the data set, ${ }^{130}$ and/or by decreasing the inelastic energy loss window $\Delta E$. This last procedure can be particularly important at low electron energies. This is illustrated in Fig. 14 which shows, for

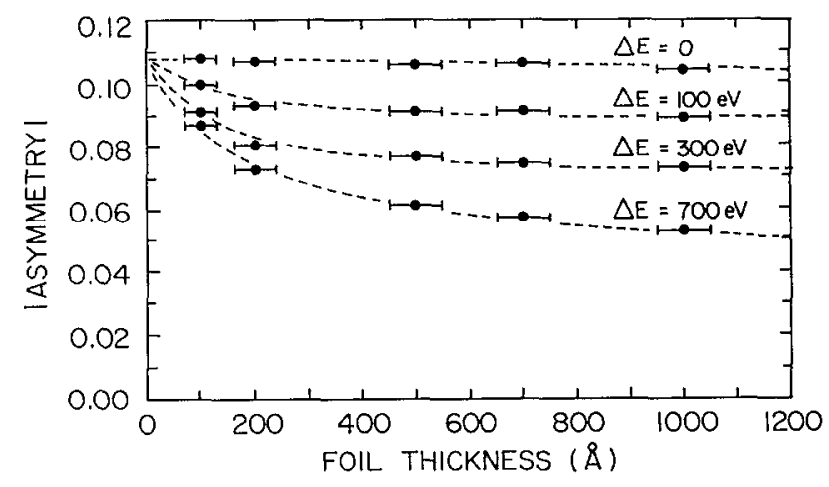

FIG. 14. Observed asymmetry $A$ for scattering of $40-\mathrm{keV}$ polarized electrons at $120^{\circ}$ as a function of (gold) target thickness for different inelastic energy loss windows $\Delta E$ (taken from Ref. 70 ).

several values of $\Delta E$, the asymmetry measured when scattering $40-\mathrm{keV}$ polarized electrons from gold targets of various thickness. ${ }^{70}$ It is apparent that a thickness extrapolation based on target thicknesses $\gtrsim 200 \AA$ with $\Delta E \sim 1 \mathrm{keV}$ could be subject to sizeable systematic error. Typically, energies $\gtrsim 100 \mathrm{keV}$, target thickness $\lesssim 200 \AA$ and energy resolutions $\Delta E \lesssim 100 \mathrm{eV}$ are required for precise measurements. Using the cylindrical retarding-potential polarimeter shown in Fig. 6 and average target thicknesses between 30 and $700 \AA$ it has recently been demonstrated that, for $E=100 \mathrm{keV}$ and $\Delta E=100 \mathrm{eV}$, the intercepts obtained using different linear fitting parameters are equal to within a relative uncertainty of $\sim 0.8 \%$, which was due almost entirely to counting statistics. ${ }^{48}$

The use of extremely thin targets raises questions concerning target morphology $48,131,132$ and its possible effect on thickness extrapolations. It is known, for example, that thin evaporated gold films tend to exhibit nucleated structure, which might significantly influence the dependence of plural scattering on average foil thickness. Although the importance of such effects is a current topic of debate, the very precise measurements just described suggest that they are relatively unimportant.

\section{Extrapolations to zero inelastic energy loss}

The high-energy resolution provided by retarding potential Mott polarimeters suggests an alternate calibration procedure. The mean rate of loss of energy with distance for an electron travelling in gold, $d E / d x$, is $\sim 1.2-0.5$ $\mathrm{eV} \AA^{-1}$ in the energy range $20-100 \mathrm{keV}$. Thus for an inelastic energy loss window $\Delta E$ of, for example, $1 \mathrm{keV}$, electrons traveling total distances $\gtrsim 1-2 \times 10^{3} \AA$ in the target foil will suffer sufficient energy loss to be discriminated against, limiting the observed scattering to the near surface region, even for bulk targets. ${ }^{41,133}$ The thickness of the region from which scattering is observed can be reduced by decreasing $\Delta E$, suggesting that an extrapolation of the observed asymmetry to zero $\Delta E$ is essentially equivalent to an extrapolation to zero foil thickness and that the data could again be normalized to the calculated Sherman function for single atom scattering in this limit. Measurements of $A$ vs $\Delta E$ are simple to carry out and thus the idea is attractive 


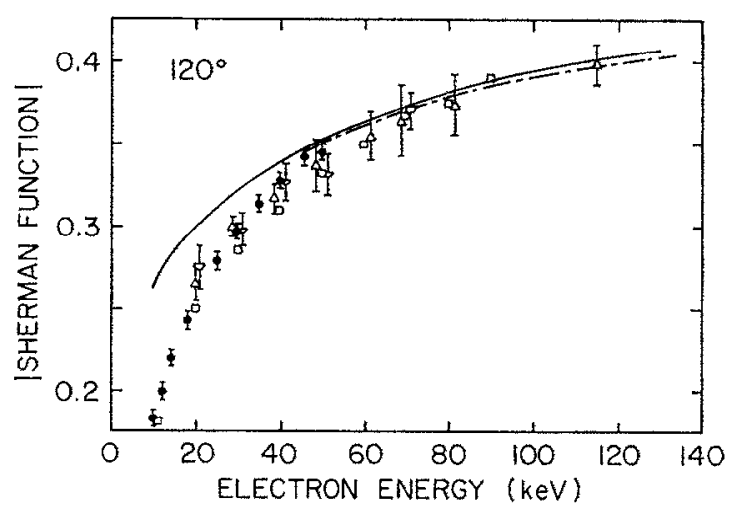

FIG. 15. Energy dependence of the Sherman function for gold at $120^{\circ}$. Theory:-, Ref. 54; -.- -, Ref. 52. Experiment: $\square$, Ref. $41(\Delta E \rightarrow 0$, $t=300 \AA) ; \Delta$, Ref. $107(\Delta E \rightarrow 0, t=1000 \AA)$; $\square$, Ref. $106(\Delta E=25 \mathrm{eV}$, $t=1250 \AA) ; 0$, Ref. $70(\Delta E \rightarrow 0, t=100 \AA)$.

from an experimental standpoint. The technique, however, must be approached with caution.

Electrons traversing high- $Z$ materials do not lose energy in a continuous fashion but rather in a series of discrete steps. ${ }^{134,135}$ Inelastic scattering is characterized by a mean free path that, for the energy range $20-120 \mathrm{keV}$, is approximately five times larger than that for elastic scattering. Thus, since inelastic scattering events tend to be associated with small scattering angles, plural elastic scattering can contribute significantly to the backscattered signal. ${ }^{133,136}$ Consequently, even complete discrimination against inelastic events does not preclude a reduction in the measured asymmetry due to plural scattering, unless extremely thin targets are used. ${ }^{48}$ Comparisons of foil thickness and inelastic energy loss extrapolations at $120 \mathrm{keV}$ suggest that plural scattering is not a problem at the $5 \%$ level. ${ }^{40}$ Below $50 \mathrm{keV}$, however, effects associated with plural scattering become more important. This is illustrated in Fig. 15 which shows calculated values of the Sherman function for $120^{\circ}$ scattering from gold together with data obtained, using relatively thick targets, by extrapolating observed scattering asymmetries to zero inelastic energy loss. It is apparent that, below $50 \mathrm{keV}$, there is an increasing discrepancy between theory and experiment. Nonetheless, the data suggest that, for energies $\gtrsim 20 \mathrm{keV}$ measurements of the asymmetry versus $\Delta E$ can, even for thick targets, provide a simple first-order calibration of a retarding-potential Mott polarimeter at the 10-20\% level. These issues are considered in detail in Ref. 48.

\section{Calculated Sherman functions}

The calibration procedures just described require normalization to calculated Sherman functions in the limit of zero target thickness or zero inelastic energy loss. A number of detailed calculations have been reported, ${ }^{52-54}$ but while each of these claims a fractional error $\$ 1.5 \%$ the discrepancies between the various calculations can amount to $4 \%$. However, for $120^{\circ}$ scattering from gold at $120 \mathrm{keV}$ the calculations agree to within $1.8 \%$. The origins of these differences are unclear, but they might stem from numer- ical errors or the choice of screening potentials. Certainly it is unlikely that the calculated values are in error by more than $3 \%$. For high-efficiency polarimeters that detect electrons scattered over a wide range of angles the Sherman function must be appropriately averaged over the collection solid angle when normalizing to theory.

Accurate calculations of Sherman functions at low electron energies ( $\leqslant 1 \mathrm{keV}$ ) are difficult due to the importance of the atomic electrons and theoretical uncertainties of $\sim 10-20 \%$ are to be expected. ${ }^{137}$ Thus, for precise measurements, polarimeters using atomic vapor beams must either be calibrated in a double-scattering experiment or by using a high-energy polarimeter. It should also be remembered that at low energies the Sherman function can change rapidly with energy and scattering angle.

\section{Measurement of $S_{\text {eff }}$ through double scattering}

Values of $S_{\text {eff }}$ can be measured directly in a doublescattering experiment. This eliminates reliance on theory but demands a high level of experimental rigor. In addition to problems associated with instrumental asymmetries and backgrounds, a stringent symmetry requirement is placed on the apparatus: the electron beam-target scattering geometry must be the same for both the first and second scattering. If this condition is satisfied it is apparent from Eqs. (6) and (8) (with $\theta_{1}=\theta_{2} \equiv \theta$ ) that determination of the asymmetry associated with the second scattering yields $S_{\text {eff }}$ directly, i.e., $A=S_{\text {eff }}^{2}$. Although double-scattering experiments have been employed to calibrate polarimeters for use in polarimetric studies, ${ }^{22,38,60,118}$ the majority of such work has centered on testing calculated Sherman functions. ${ }^{10,45-47,61-66}$ With detailed experimental analysis and careful use of monitor counters to eliminate instrumental asymmetries, ${ }^{46,47}$ it is now possible to measure values of $S_{\text {eff }}$ to within a fractional error of $\sim 0.2 \%$, which represents the state-of-the-art in accuracy for Mott polarimetry. However, while measurements of $S_{\text {eff }}$ at this level of accuracy can be accomplished, values of the Sherman function derived from double-scattering experiments are still subject to energy and thickness extrapolations. These introduce an additional uncertainty of $\sim 3 \%$ which is comparable to the discrepancies between the various calculated values. $^{47}$

\section{Direct calibration using electrons of known polarization}

Values of $S_{\text {eff }}$ can also be measured directly by using an input beam of known polarization and observing the resultant scattering asymmetry. This eliminates the need for involved extrapolation procedures and holds the promise of providing improved polarimetric accuracies in the future. Initial experiments of this type used electrons produced in $\beta$ decay whose polarization was assumed to equal $v / c$. Much of this work, however, focused on obtaining Sherman functions for comparison with theoretical values. ${ }^{19,67,68}$ Although the data suffer from depolarization effects due to plural and multiple scattering in both the target and $\beta$ source, they are in qualitative agreement with 
theory. Recently, the polarization of electrons produced by a number of $\beta$ emitters has been determined using a Mott polarimeter calibrated with electrons produced in the $\beta$ decay of ${ }^{60} \mathrm{Co}^{105}$ The principal uncertainty in such measurements stems from depolarization in the source itself, which must be corrected for. Instrumental asymmetries must also be carefully monitored, however, to ensure that the various sources yield beams with similar electron-optical properties. With such precautions, measurements of relative $\beta$-ray polarizations to within $\sim 1 \%$ appear feasible, although the absolute accuracy is limited by the uncertainty in the polarization of the calibration standard. For ${ }^{60} \mathrm{Co}$, which has been the subject of the most precise investigations, the polarization has been measured to $\sim 1.5 \%{ }^{23}$ While this calibration technique is of obvious value in $\beta$ ray studies, the problems associated with handling radioactive materials and the dimness of the sources diminishes its attractiveness in other applications.

Electrons of accurately known polarization can also be obtained via chemiionization reactions of the type

$$
\begin{aligned}
& \operatorname{He}\left(2^{3} S\right)\{\uparrow \uparrow\}+X Y\{\uparrow \uparrow\} \\
& \quad \rightarrow \operatorname{He}\left(1^{1} S\right)\{\uparrow \uparrow\}+X Y^{+}\{\uparrow\}+e^{-}\{\uparrow\},
\end{aligned}
$$

involving spin-polarized $\mathrm{He}\left(2^{3} S\right)$ metastable atoms and a spin-singlet target. ${ }^{138}$ The polarization of the liberated electrons is equal to that of the metastable atoms, which can be measured directly using a Stern-Gerlach analyzer. Polarized electrons obtained in this manner with crossed metastable atom and target beams have been used to calibrate a compact retarding-potential polarimeter. ${ }^{69}$ The calibration uncertainty of $\sim 5 \%$ results from difficulties associated with measuring the metastable atom polarization and from secondary electron ejection caused by scattered metastable atoms that strike chamber surfaces. It is reasonable to expect that both sources of uncertainty can be substantially reduced in the future.

Direct calibration has also been undertaken using electrons from a GaAs source whose polarization was accurately determined using an optical technique that involves observation of the radiation emitted by an atomic target excited by the electron beam. ${ }^{70,139,140}$ If excitation occurs through an exchange process, the excited states will have a net orientation relative to the quantization axis defined by the incident electron polarization that, upon subsequent decay, will be manifest through circular polarization of the emitted radiation. Thus by measuring the polarization of the emitted radiation it is, in principle, possible to determine the polarization of the incident electrons. This approach was first successfully demonstrated using zinc atoms and, later, with mercury. ${ }^{141,142}$ Heavy metal targets are, however, not ideal for absolute polarimetry because the expressions that relate the degree of circular polarization of the emitted radiation to the incident electron polarization must include the effects of hyperfine depolarization and of the presence of various isotopes. Failure of LS coupling must also be considered, as must negative ion resonances. The use of helium as the target, however, eliminated these problems and it is possible to accurately measure the polarization of an electron beam by observing the polarization of the $3889 \AA 3^{3} P \rightarrow 2^{3} S$ radiation emitted following excitation, provided that the electron energy lies between $23.0 \mathrm{eV}$ (the threshold for $1^{1} S \rightarrow 3^{3} P$ excitation) and $23.6 \mathrm{eV}$ (where cascading from the $4^{3} S$ level can begin to affect the measurements). A helium polarimeter has been used to calibrate a compact retarding-potential Mott analyzer. ${ }^{70}$ The resulting fractional uncertainty in $S_{\text {eff }}$ of $\sim 0.8 \%$ was dominated by systematic errors associated with measurement of the polarization of the emitted radiation. With care, however, optical polarization measurements can be made to $\sim 0.1 \%$ and experiments are now underway at Rolla to determine if subsequent polarimeter calibrations with uncertainties $\lesssim 0.2 \%$ can be achieved. This is an accuracy level comparable to the best doublescattering measurements. Comparison of the two methods at this level would result in the most accurate measurements of electron polarization yet made.

A GaAs source has also been used in a novel calibration procedure which involves diffraction at a $\mathrm{Pt}(111)$ surface. ${ }^{143}$ Detailed analysis reveals that, for specular diffraction under conditions where the scattering plane is a mirror plane in the surface, the value of $S_{\text {eff }}$ appropriate to a polarimeter can be obtained by measuring the spin-orbit induced scattering asymmetry at the target surface and the polarization of the incident and scattered electrons using the polarimeter. Using this technique $S_{\text {eff }}$ for a conventional Mott polarimeter has been determined to within an uncertainty $\sim 2 \%$.

\section{DISCUSSION}

In this section the factors important in selecting a polarimeter for a specific application are discussed. Typical operating characteristics for the different types of Mott polarimeter are listed in Table I.

One important parameter to be considered is the electron-optical quality of the electron beam to be analyzed. For any paraxial electron optical system in which electron current is conserved, the law of Helmholtz-Lagrange ${ }^{144}$ states that for any positions 1 and 2 along the beam there is a conservation of the product of the energy $E$, crosssectional area $C$, and solid angle $\Omega$, i.e.,

$$
E_{1} C_{1} \Omega_{1}=E_{2} C_{2} \Omega_{2} \text {. }
$$

The electron optical acceptance, EC , for high-energy Mott polarimeters is typically quite large, $\sim 10^{3}-10^{4}$ $\mathrm{mm}^{2} \mathrm{sr} \mathrm{eV}$, whereas for low-energy polarimeters it is small, $\lesssim 10^{2} \mathrm{~mm}^{2} \mathrm{sr} \mathrm{eV}$. ${ }^{44}$ Thus only electron beams of good optical quality, i.e., having small cross-sectional area and divergence, can be coupled into a low-energy polarimeter without a major loss in beam intensity. Low-energy polarimeters also require an input beam with a narrow energy spread because low-energy Mott scattering is sensitive to energy. Both constraints are relaxed for high-energy polarimeters, although the energy spread of the input beam could be important for a retarding-potential polarimeter if small inelastic energy loss windows $\Delta E$ are employed.

The required polarimetric accuracy and efficiency are also important. If efficiency is of paramount concern, such 
TABLE I. Comparison of Mott polarimeters.

\begin{tabular}{|c|c|c|c|c|c|c|c|}
\hline Type & $\begin{array}{l}\text { Operating } \\
\text { energy }\end{array}$ & Target & $\begin{array}{c}E C \Omega \\
\left(\mathrm{mm}^{2} \mathrm{sr} \mathrm{eV}\right)\end{array}$ & $\Delta E$ & $\begin{array}{l}\text { Sensitivity } \\
\qquad I / I_{0}\end{array}$ & $\left|S_{\mathrm{eff}}\right|$ & $\begin{array}{c}\text { Efficiency } \\
(\epsilon)\end{array}$ \\
\hline $\begin{array}{l}\text { Conventional } \\
\text { Retarding }\end{array}$ & $90-300 \mathrm{keV}$ & gold foil & $\sim 10^{3}$ & $\sim 10 \mathrm{keV}$ & $\sim 10^{-2}-10^{-3}$ & $0.2-0.4$ & $1 \times 10^{4}$ \\
\hline $\begin{array}{l}\text { potential } \\
\text { (cylindrical) }\end{array}$ & $20-120 \mathrm{keV}$ & gold foil & $-10^{4}$ & $0-1.5 \mathrm{keV}$ & $\sim 10^{-6}-10^{-7}$ & $0.2-0.4$ & $10^{-7 a}$ \\
\hline $\begin{array}{l}\text { Retarding } \\
\text { potential } \\
\text { (spherical) }\end{array}$ & $10-50 \mathrm{keV}$ & $\begin{array}{l}\text { gold foil } \\
\text { or solid }\end{array}$ & $\sim 10^{4}$ & $0-1.5 \mathrm{keV}$ & $-10^{-3}-10^{-4}$ & $0.15-0.35$ & $2 \times 10^{-5 a}$ \\
\hline $\begin{array}{l}\text { Retarding } \\
\text { potential } \\
\text { (conical) }\end{array}$ & $10-35 \mathrm{keV}$ & $\begin{array}{l}\text { gold foil } \\
\text { or solid }\end{array}$ & $\sim 10^{4}$ & $0-1.5 \mathrm{keV}$ & $\sim 10^{-2}-10^{-4}$ & $0.1-0.25$ & $3 \times 10^{-5 a}$ \\
\hline $\begin{array}{l}\text { Diffuse } \\
\text { scattering } \\
\text { Mercury }\end{array}$ & $150 \mathrm{eV}$ & gold solid & $-10^{z}$ & $-40 \mathrm{eV}$ & $10^{-1}-10^{-2}$ & $\sim 0.1$ & $2 \times 10^{-4}$ \\
\hline yapor & $10-1000 \mathrm{eV}$ & mercury vapor & & $\sim 5 \mathrm{eV}$ & $10^{-4}-10^{-5}$ & $0.1-0.4$ & $4 \times 10^{-5}$ \\
\hline
\end{tabular}

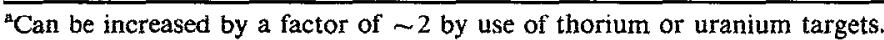

as might be the case when the electrons to be analyzed result from some gas-phase collision process, the use of a conventional high-energy or diffuse scattering polarimeter is advantageous. If, however, the beam polarization is small the effects of systematic errors due to, for example, instrumental asymmetries must be minimized. Sensitivity to such errors is reduced if $S_{\text {eff }}$ is large, suggesting that the use of a conventional polarimeter is to be preferred, although if the input current is sufficient, use of a highenergy retarding-potential polarimeter, possibly with a uranium or thorium target, should be considered. Retardingpotential analyzers also have the advantage that it is possible to increase $S_{\text {eff }}$, at the expense of a reduced efficiency, simply by decreasing the inelastic energy loss window $\Delta E$. If high precision measurements are required, without the added complexity of calibrating the polarimeter by optical or double-scattering methods, the best approach is to use a high-energy retarding-potential polarimeter calibrated at small $\Delta E$ by use of a target thickness extrapolation. ${ }^{48}$

The choice of polarimeter may also be dictated by physical constraints. Space requirements may rule out large high voltage polarimeters. For example, some experiments require a compact polarimeter to undertake in situ angle-resolved polarization measurements. The conical retarding-potential, low-energy diffuse scattering, and mercury vapor polarimeters are all compact and offer reasonable efficiencies, although mercury vapor polarimeters are not readily compatible with UHV operation.

Electron polarizations can also be measured using techniques other than Mott scattering. For example, as discussed previously, optical electron polarimeters have been developed that involve photon emission from targets excited by the incident electron beam. These offer high accuracy, but their efficiencies are rather low. At very high electron energies ( $\gtrsim 1 \mathrm{MeV}$ ), where Mott scattering cross sections become very small, polarimeters based on electron-electron (Moller) scattering are frequently employed. ${ }^{35,36}$ In such instruments, the scattering asymmetry that results when longitudinally polarized electrons are scattered by polarized electrons in a ferromagnetic foil is measured. The scattering asymmetry is, however, limited by the low average polarization of the electrons in the foil and is typically smaller than that for Mott scattering. Other very-high-energy techniques that have been used are based on electron-photon coupling and involve bremsstrahlung production by longitudinally polarized electrons with subsequent measurement of the gamma-ray circular polarization, ${ }^{35,36}$ determination of the azimuthal dependence of bremsstrahlung produced by transversely polarized electrons, ${ }^{145,146}$ and measurement of Compton scattering asymmetries in collisions between circularly polarized visible photons and highly relativistic longitudinally polarized electrons. ${ }^{147}$ Electron-photon polarimeters are characterized by relatively low asymmetries and cross sections, and are difficult to implement.

Polarimeters based on low-energy electron diffraction (LEED) and secondary-electron emission from solids have also been implemented. These offer compactness and high efficiency but, because they employ incident electron energies of $\sim 100 \mathrm{eV}$, their performance is sensitive to the target surface conditions. Thus, the target surfaces must be renewed or cleaned periodically and maintained in a UHV environment, and toutine calibration checks are required. The LEED polarimeter is based on electron diffraction from a single crystal (most often tungsten ${ }^{27,79,148,149}$ ). The left-right asymmetry in the Bragg peaks that results from the spin-orbit interaction is proportional to the electron polarization and provides a measure of the input beam polarization. The scattering asymmetry is, however, sensitive to both angle of incidence and energy requiring an input beam with a narrow spread in both energy and angle. A polarimeter based on spin dependences in low-energy $(\sim 10 \mathrm{eV})$ electron scattering from a magnetized iron surface has also been demonstrated that offers high efficiency. ${ }^{150}$

Secondary electron polarimeters are based on the observation that the net current absorbed by a metallic target on which an electron beam impinges (i.e., the difference between the incident current and the ejected secondary electron current) is spin sensitive, a phenomenon due to exchange and/or spin-orbit effects. When operating at an 
energy for which the net absorbed current is near zero, the absorbed current can depend quite dramatically on the incident beam polarization. This effect has been observed with ferromagnetic targets, ${ }^{151}$ and with nonmagnetic polycrystalline $^{152}$ and crystalline $e^{153,154}$ targets. Absorbed current polarimeters are sensitive to small changes in absolute values of polarization, but can only be used in an analog mode. In addition, they are very sensitive to the angle of the incident beam ${ }^{154}$ and are thus perhaps better suited to monitoring the polarization of a (stable) beam than to measuring its absolute polarization.

As evident from the above discussions, Mott polarimeters are attractive for use in any application requiring electron spin polarimetry. They provide good efficiency and analyzing power, they are relatively simple to implement experimentally, they are not affected by many of the problems associated with other types of polarimeters, and the physical basis of their operation is well understood.

\section{ACKNOWLEDGMENTS}

The research by the authors and their colleagues described in this paper is supported by the National Science Foundation and the Robert A. Welch Foundation.

' G. E. Uhlenbeck and S. A. Goudsmit, Naturwiss. 13, 953 (1925); see also Physics Today 29, No. 6, 40 (1976).

${ }^{2}$ W. Pauli, Z. Phys. 43, 601 (1927).

${ }^{3}$ P. A. M. Dirac, Proc. R. Soc. A 117, 610 (1928).

${ }^{4}$ N. F. Mott, Proc. R. Soc. A 124, 425 (1929).

${ }^{3}$ N. F. Mott, Proc. R. Soc. A 135, 429 (1932).

'In this regard, however, see H. Dehmelt, Science 247, 539 (1990).

${ }^{7}$ H. A. Tolhoek, Rev. Mod. Phys. 28, 277 (1956).

J. Kessler, Rev. Mod. Phys. 41, 3 (1969).

${ }^{9}$ C. G. Shull, C. T. Chase, and F. E. Myers, Phys. Rev. 63, 29 (1943).

${ }^{10}$ W. H. Louisell, R. W. Pidd, and H. R. Crane, Phys. Rev. 91, 475 (1953); 94, 7 (1954).

"C. S. Wu, E. Ambler, R. W. Hayward, D. D. Hoppes, and R. P. Hudson, Phys. Rev, 105, 1431 (1957).

${ }^{12}$ T. D. Lee and C. N. Yang, Phys. Rev. 105, 1671 (1957); L. Landau, Nucl. Phys. 3, 127 (1957); R. B. Curtis and R. R. Lewis, Phys. Rev. 107, 543 (1957); J. D. Jackson, S. B. Treiman, and H. W. Wyld, Jr., Nucl. Phys. 4, 206 (1957).

${ }^{13} \mathrm{H}$. Fraunfelder, R. Bobone, E. von Goeler, N. Levine, H. R. Lewis, R. N. Peacock, A. Rossi, and G. DePasquali, Phys. Rev. 106, 386 (1957).

${ }^{14}$ A. I. Alikhanov, G. P. Eliseiev, V. A. Liubimov, and B. V. Ershler, Nucl. Phys. 5, 588 (1958).

${ }^{15}$ A. I. Alikhanov, G. P. Eliseiev, and V. A. Lubimov, Nucl. Phys. 7, 655 (1958), and references therein.

${ }^{16}$ P. E. Spivak and L. A. Mikaelian, Nucl. Phys. 20, 475 (1960), and references therein.

${ }^{17}$ J. S. Greenberg, D. P. Malone, R. L. Gluckstern, and V. W. Hughes, Phys. Rev. 120, 1393 (1960), and references therein

${ }^{18}$ A. R. Brosi, A. I. Galonsky, B. H. Ketelle, and H. B. Willard, Nucl. Phys. 33, 353 (1962), and references therein.

${ }^{19} \mathrm{~V}$. Eckardt, A. Ladage, and U. V. Moellendorff, Phys. Lett. 13, 53 (1964), and references therein.

${ }^{20} \mathrm{H}$. Beekhuis, P. Boskma, J. van Klinken, and H. de Waard, Nucl. Phys. 79,220 (1966), and references therein.

${ }^{21}$ D. M. Lazarus and J. S. Greenberg, Phys. Rev. D 2, 45 (1970), and references therein.

${ }^{22}$ F. W. J. Koks and J. van Klinken, Nucl. Phys. A 272, 61 (1976), and references therein.

${ }^{23} \mathrm{~J}$. van Klinken, F. W. J. Koks, and H. Behrens, Phys. Lett. B 79, 199 (1978).

${ }^{24}$ See, e.g., D. T. Pierce, R. J. Celotta, G.-C. Wang, W. N. Unertl, A. Galejs, C. E. Kuyatt, and S. R. Mielczarek, Rev. Sci. Instrum. 51, 478 (1980).
${ }^{25}$ J. Kessler, Polarized Electrons, 2nd ed. (Springer, Berlin, 1985).

${ }^{26}$ See, e.g., G. Baum, M. Fink, W. Raith, H. Steidl, and J. Taborski, Phys. Rev. A 40, 6734 (1989), and references therein.

${ }^{27}$ See, e.g., Polarized Electrons in Surface Physics, edited by R. Feder (World Scientific, Singapore, 1985).

${ }^{28}$ J. L. Robins, R. J. Celotta, J. Unguris, D. T. Pierce, B. T. Jonker, and G. A. Prinz, Appl. Phys. Lett. 52, 1918 (1988); K. Koike, H. Matsuyama, K. Mitsuoka, and K. Hayakawa, Jpn. J. Appl. Phys. 25, L758 (1986).

${ }^{29}$ D. L. Abraham and H. Hopster, Phys. Rev. Lett. 62, 1157 (1989).

${ }^{30}$ See, e.g., C. Y. Prescott et al., Phys. Lett. B 77, 347 (1978).

${ }^{31}$ See, e.g., D. I. Lowenstein, in High-Energy Spin Physics, edited by K. J. Heller (American Institute of Physics, New York, 1989), AIP Conference Proc. No. 187.

${ }^{32}$ P. S. Cooper et al., Phys. Rev. Lett. 42, 1386 (1979).

${ }^{33}$ P. S. Farago, Adv. Electron. Phys. 21, 1 (1965).

${ }^{34}$ P. S. Farago, Rep. Prog. Phys. 34, 1055 (1971).

${ }^{35} \mathrm{H}$. Frauenfelder and A. Rossi, in Methods of Experimental Physics, edited by L. C. Yuan and C. S. Wu (Academic, New York, 1963), Vol. $5 \mathrm{~B}$.

${ }^{36} \mathrm{H}$. Frauenfelder and R. M. Steffan, in Alpha, Beta and Gamma Ray Spectroscopy, edited by K. Sieghahn (North Holland, Amsterdam, 1965).

${ }^{37}$ H. F. Schopper, Weak Interactions and Nuclear Beta Decay (North Holland, Amsterdam, 1966).

${ }^{38}$ J. van Klinken, Nucl. Phys. 75, 161 (1966), and references therein.

${ }^{39}$ K. Jost, F. Kaussen, and J. Kessler, J. Phys. E 14, 735 (1981).

${ }^{40}$ L. A. Hodge, T. J. Moravec, F. B. Dunning, and G. K. Walters, Rev. Sci. Instrum. 50, 5 (1979).

${ }^{41}$ D. M. Campbell, C. Hermann, G. Lampel, and R. Owen, J. Phys. E 18, 664 (1985).

${ }^{42}$ G. D. Fletcher, T. J. Gay, and M. S. Lubell, Phys. Rev. A 34, 911 (1986).

${ }^{43}$ D. T. Pierce, R. J. Celotta, M. H. Kelley, and J. Unguris, Nucl. Instrum. Methods, A 266, 550 (1988).

${ }^{44}$ J. Unguris, D. T. Pierce, and R. J. Celotta, Rev. Sci. Instrum. 57, 1314 (1986).

${ }^{45}$ L. Mikaelyan, A. Borovoi, and E. Denisov, Nucl. Phys. 47, 328 (1963), and references therein.

${ }^{46}$ A. Gellrich, K. Jost, and J. Kessler, Rev, Sci. Instrum. 61, 3399 (1990).

${ }^{47}$ A. Gellrich and J. Kessler, Phys. Rev. A 43, 204 (1991).

${ }^{48}$ T. J. Gay, M. A. Khakoo, J. A. Brand, J. E. Furst, W. V. Meyer, W. M. K. P. Wijayaranta, and F. B. Dunning, Rev. Sci. Instrum. (in press).

${ }^{49}$ L. H. Thomas, Nature 117, 514 (1926).

${ }^{50}$ N. Sherman, Phys. Rev. 103, 1601 (1956); see also N. Sherman and D. F. Nelson, Phys. Rev. 114, 1541 (1959); S.-R. Lin, N. Sherman, and J. K. Percus, Nucl. Phys. 45, 492 (1963).

${ }^{51}$ J. W. Motz, H. Olsen, and H. W. Koch, Rev. Mod. Phys. 36, 881 (1964).

${ }^{52}$ G. Holzwarth and H. J. Meister, Nucl. Phys. 59, 56 (1964).

${ }^{53}$ W. Bühring, Z. Phys. 208, 286 (1968); 212, 61 (1968).

${ }^{54}$ A. W. Ross and M. Fink, Phys. Rev. A 38, 6055 (1988).

${ }^{55}$ H. S. W. Massey and C. B. O. Mohr, Proc. Roy. Soc. (London) A 177, 341 (1941).

${ }^{56}$ B. Awe, F. Kemper, F. Rosicky, and R. Feder, J. Phys. B 16, 603 (1983).

${ }^{57}$ R. Haberland and L. Fritsche, J. Phys. B 20, 121 (1987).

${ }^{58}$ K. Hasenburg, D. H. Madison, K. Bartschat, and K. Blum, J. Phys. B 19, 1803 (1986); R. P. McEachran and A. D. Stauffer, J. Phys. B 19, 3523 (1986).

${ }^{59}$ A. W. Ross and M. Fink, Phys. Rev. A 38, 6055 (1988); D. Gregory and M. Fink, Atomic and Nuclear Data Tables 14, 39 (1974); M. Fink and J. Ingram, Atomic Data 4, 129 (1972); M. Fink and J. Yates, Atomic Data 1, 385 (1970).

${ }^{60}$ H. Deichsel, Z. Phys. 164, 156 (1961).

${ }^{61}$ V. A. Apalin, I. Ye. Kutikov, I. I. Lukashevich, L. A. Mikaelyan, G. V. Smirnov, and P. Ye. Spivak, Nucl. Phys. 31, 657 (1962).

${ }^{62}$ H. Boersch, R. Schliepe, and K. E. Schrief, Nucl. Phys. A 163, 625 (1971).

${ }^{63}$ N. Ryu, J. Phys. Soc. Jpn. 7, 125, 130 (1952); 8, 575, 804 (1953).

${ }^{64}$ W. Pettus, Phys. Rev. 109, 1458 (1958). 
${ }^{65}$ D. F. Nelson and R. W. Pidd, Phys. Rev. 114, 728 (1959).

${ }^{66}$ P. E. Spivak, L. A. Mikaelyan, I. E. Kutikov, V. F. Apalin, I. I. Lukashevich, and G. V. Smirnov, Sov. Phys. JETP 14, 759 (1962), and references thercin.

${ }^{67} \mathrm{H}$. Bienlein, G. Felsner, K. Gunther, H. von Issendorff, and $\mathrm{H}$. Wegener, Z. Phys. 154, 376 (1959).

${ }^{68}$ H. Bienlein, G. Felsner, R. Fleischmann, K. Gunther, H. von Issendorff, and H. Wegener, Z. Phys. 155, 101 (1959).

${ }^{69}$ D. M. Oro, W. H. Butler, F. B. Dunning, and G. K. Walters, Rev. Sci. Instrum. 62, 667 (1991).

${ }^{70}$ M. Uhrig, A. Beck, J. Goeke, F. Eschen, M. Sohn, G. F. Hanne, K. Jost, and J. Kessier, Rev. Sci. Instrum. 60, 872 (1989).

${ }^{71}$ E. Kisker, G. Baum, A. H. Mahan, W. Raith, and B. Reihl, Phys. Rev. B 18, 2256 (1978).

${ }^{72}$ K. Jost and J. Kessler, 7. Phys. 195, 1 (1966).

${ }^{73}$ J. Kessler, J. Lorenz, H. Rempp, and W. Bühring, Z. Phys. 246, 348 (1971).

${ }^{74}$ G. F. Hanne and J. Kessler, J. Phys, B 9, 791 (1976)

${ }^{75}$ U. Heinzmann, J. Phys. B 11, 399 (1978).

${ }^{76}$ R. Möllenkamp, W. Wübker, O. Berger, K. Jost, and J. Kessler, J, Phys. B 17, 1107 (1984).

${ }^{77}$ M. Campagna, D. T. Pierce, F. Meier, K. Sattler, and H. C. Siegmann, Adv. Electron. Electron Phys. 41, 113 (1976); B. Reihl, M. Erbudak, and D. M. Campbell, Phys. Rev. B 19, 6358 (1979).

${ }^{78}$ M. Landolt, R. Allenspach, and D. Mauri, J. Appl. Phys. 57, 3626 (1985)

${ }^{79}$ M. R. O'Neill, M. Kalisvaart, F. B. Dunning, and G. K. Walters, Phys. Rev. Lett. 34, 1167 (1975)

${ }^{80}$ M. Kalisvaart, M. R. O'Neill, T. W. Riddle, F. B. Dunning, and G. K. Walters, Phys. Rev. B 17, 1570 (1978)

${ }^{81}$ E. Kisker, R. Clauberg, and W. Gudat, Rev. Sci. Instrum. 53, 1137 (1982).

${ }^{82}$ R. Raue, H. Hopster, and E. Kisker, Rev. Sci. Instrum. 55, 383 (1984).

${ }^{83}$ C. K. Sinclair, E. L. Garwin, R. H. Miller, and C. Y. Prescott, in High Energy Physics with Polarized Beams and Targets, edited by M. L. Marshak (AIP, New York, 1976), AIP Conf. Proc. No. 35.

${ }^{84}$ M. J. Alguard, J. E. Clendenin, R. D. Ehrlich, V. W. Hughes, J. S. Ladish, M. S. Lubell, K. P. Schüler, G. Baum, W. Raith, R. H. Miller, and W. Lysenko, Nucl. Instrum. Methods, 163, 29 (1979).

${ }^{85}$ T. Nakanishi, K. Dohmae, S. Fukui, Y. Hayashi, I. Hirose, N. Horikawa, T. Ikoma, Y. Kamiya, M. Kurashina, and S. Okumi, Jpn. J. Appl. Phys, 25, 766 (1986).

${ }^{86}$ N. Ludwig, A. Bauch, P. Nass, E. Reichert, and W. Welker, Z. Phys. D 4. 177 (1986).

${ }^{87}$ D. Hils and H. Kleinpoppen, J. Phys. B 11, L283 (1978).

${ }^{88}$ V. W. Hughes, R. L. Long, Jr, M. S. Lubell, M. Posner, and W. Raith, Phys. Rev. A 5, 195 (1972).

${ }^{89}$ P. F. Wainwright, M. J. Alguard, G. Baum, and M. S. Lubell, Rev. Sci. Instrum. 49, 571 (1978)

${ }^{90}$ S. F. Alvarado, F. Ciccacci, and M. Campagna, Appl. Phys. Lett. 39, 615 (1981)

${ }^{91}$ K. Koike and K. Hayakawa, Jpn. J. Appl. Phys. 22, 1332 (1983).

${ }^{92}$ W. Hartmann, D. Conrath, W. Gasteyer, H. J. Gessinger, W. Heil, H. Kessler, L. Koch, E. Reichert, H. G. Andresen, T. Kettner, B. Wagner, J. Ahrens, J. Jethwa and F. P. Schäfer, Nucl. Instrum. Meth. A 286, 1 (1990).

${ }^{93}$ H. Hopster, Phys. Rev. B 36, 2325 (1987).

${ }^{94}$ W. Wübker, R. Möllenkamp, and J. Kessler, Phys. Rev, Lett. 49, 272 (1982).

${ }^{95}$ See, e.g., F. Gürsey, Phys. Rev. 107, 1734 (1957), and references therein.

${ }^{96}$ A. de-Shalit, S. Kuperman, H. J. Lipkin, and T. Rothem, Phys. Rev. 107, 1459 (1957)

${ }^{97}$ W. Bühring and J. Heintze, Z. Phys. 153, 237 (1958).

${ }^{98}$ P. E. Cavanagh, Proc. Roy. Soc. 246, 466 (1958).

${ }^{99}$ S. Cuperman, Nucl. Phys. 28, 84 (1961).

${ }^{100}$ R. Sosnowski, Z. Wilhelmi, and J. Wojtkowska, Nucl. Phys. 26, 280 (1961).

${ }^{101}$ R. O. Avakyan, G. L. Bayatyan, M. E. Vishnevskii, and E. V. Pushkin, Sov. Phys. JETP 14, 491 (1962).

102 J. van Klinken, Nucl. Phys. 75, 145 (1966).

${ }^{103} \mathrm{H}$. Wenninger, J. Stiewe, H. Muusz, and H. Leutz, Nucl. Phys. A 96, 177 (1967)

${ }^{104}$ J. P. Ribeiro and J. Byrne, Z. Phys. A 298, 301 (1980),
${ }^{105}$ R. Gauder, E. Speller, U. Zierer, O. Boslau, A. Hilscher, and K.-W. Hoffman, Z. Phys. A. 330, 423 (1988).

${ }^{106}$ J. J. McClelland, M. R. Scheinfein, and D. T. Pierce, Rev, Sci. Instrum. 60, $683(1989)$

${ }^{107}$ L. G. Gray, M. W. Hart, F. B. Dunning, and G. K. Walters, Rev. Sci. Instrum. 55, 88 (1984).

${ }^{108}$ F, B. Dunning, L. G. Gray, J. M. Ratliff, F.-C. Tang, X. Zhang, and G. K. Walters, Rev. Sci. Instrum. 58, 1706 (1987).

${ }^{109}$ F. C. Tang, X. Zhang, F. B. Dunning, and G. K. Walters, Rev, Sci. Instrum. 59, 504 (1988)

${ }^{110}$ D. P. Pappas and H. Hopster, Rev. Sci. Instrum. 60, 3068 (1989).

${ }^{111}$ C. Ranganathaiah, J. L. Robins, A. L. Yates, W. C. Macklin, R. A. Anderson, and J. F. Williams, J. Electron Spectros. Rel. Phenom. 51, 331 (1990)

${ }^{112}$ F. B. Dunning, F. C. Tang, and G. K. Walters, Rev. Sci. Instrum. 58, $2195(1987)$.

${ }^{113}$ X. Zhang, H. Hsu, F. B. Dunning, and G. K. Walters, J, Vac. Sci. Technol. A 9, 1932 (1991).

${ }^{114}$ G. A. Mulhollan, Xia Zhang, F. B. Dunning, and G. K. Walters, Phys. Rev. B. 41, 8122 (1990)

${ }^{115}$ M. R. Scheinfein, D. T. Pierce, J. Unguris, J. J. McClelland, R. J. Celotta, and M. H. Kelley, Rev. Sci. Instrum. 60, 1 (1989).

${ }^{110}$ M. R. Scheinfein, J. Unguris, M. H. Kelley, D. T. Pierce, and R. J Celotta, Rev. Sci. Instrum. 61, 2501 (1990).

${ }^{117}$ J. Woods, M. Tobise, and R. C. O'Handley, Rev. Sci. Instrum. 60, 688 (1989).

${ }^{138}$ H. Deichsel and E. Reichert, Z. Phys. 185, 169 (1965).

${ }^{119}$ H. Deichsel, E. Reichert, and H. Steidl, Phys. Lett. 18, 284 (1965).

${ }^{120}$ H. Deichsel, E, Reichert, and H. Steidl, Z. Phys. 189, 212 (1966).

${ }^{121}$ M. Düweke, N. Kirchner, E. Reichert, and S. Schön, J. Phys. B 9, 1915 (1976).

${ }^{122}$ R. Loth, Z. Phys. 203, 66 (1967)

${ }^{123}$ W. Eckstein, Z. Phys. 203, 59 (1967).

${ }^{124}$ W. Gehenn, R. Haug, M. Wilmers, and H. Deichsel, Z. Angew, Physik 28, 142 (1969)

${ }^{125}$ K. Jost, F. Kaussen, and J. Kessler, J. Phys. E 14, 735 (1981).

${ }^{126} \mathrm{R}$. E. Scholten, J. J. McClelland, M. H. Kelley, and R. J. Celotta, Rev, Sci. Instrum. 59, 506 (1988).

${ }^{127}$ M. E. Rose and H. A. Bethe, Phys. Rev, 55, 277 (1939)

${ }^{128} \mathrm{H}$. Wegener, Z, Phys. 151, 252 (1958).

${ }^{129}$ V. Hnizdo, Nucl. Instrum. Methods 109, 503 (1973).

${ }^{130}$ For a discussion of methods used to measure film thickness see W. A. Pliskin and S. J. Zanin, in Handbook of Thin Film Technology, edited by L. I. Maissel and R. Glang (McGraw-Hill, New York, 1970), Chap. 11 .

${ }^{131}$ L. L. Kazmerski and D. M. Racine, J. Appl. Phys, 46, 791 (1975).

${ }^{132}$ K. L. Chopra, Thin Film Phenomena (McGraw-Hill, New York, 1969), Chap. 4.

${ }^{133}$ O. C. Wells, Scanning Electron Microscopy (McGraw-Hill, New York, 1974), Chap. 3.

${ }^{134}$ See, for example, M. R. Sogard, I. Appl. Phys. 51, 4417 (1980).

${ }^{135}$ R. F. Dashen, Phys. Rev. A 134, 1025 (1964).

${ }^{136}$ R. E. Burge and G. H. Smith, Proc. Phys. Soc. London 79, 673 (1962).

${ }^{137}$ D. H. Madison (private communication).

${ }^{138}$ P. J. Keliher, F, B. Dunning, M. R. O'Neill, R. D. Rundel, and G. K. Walters, Phys. Rev. A 11, 1271 (1975).

${ }^{139}$ T. J. Gay, J. Phys. B 16, L553 (1983)

${ }^{140}$ J. Goeke, J. Kessler, and G. F. Hanne, Phys. Rev. Letl. 59, 1413 (1987).

${ }^{141}$ M. Eminyan and G. Lampel, Phys. Rev, Lett. 45, 1171 (1980)

${ }^{142}$ A. Wolcke, K. Bartschat, K. Blum, H. Borgmann, G. F. Hanne, and J. Kessler, J. Phys. B 16, 639 (1983).

${ }^{143} \mathrm{H}$. Hopster and D. L. Abraham, Rev. Sci. Instrum. 59, 49 (1988).

${ }^{144}$ See, e.g., J. H. Moore, C. C. Davis, and A. M. Coplan, Building Scientific Apparatus, 2nd ed. (Addison-Wesley, Redwood City, 1989).

${ }^{145}$ A. Aehlig, Z. Phys. A 294, 291 (1979).

${ }^{146}$ H. R. Schaefer, W. von Drachenfels, and W. Paul, Z. Phys. A 395, 213 (1982). 
${ }^{147}$ H. D. Bremer, H. C. Dehne, H. C. Lewin, H. Mais, R. Neumann, R. Rossmanith, and R. Schmidt, in High Energy Physics with Polarized Beams and Polarized Targets, edited by C. Joseph and J. Soffer (Birkhäuser, Basel, 1981).

${ }^{148} \mathrm{~J}$. Kirschner, Polarized Electrons at Surfaces, (Springer, Berlin, 1985), Springer Tracts in Modern Physics, Vol. 106.

${ }^{149} \mathrm{~J}$. Kirschner and R. Feder, Phys. Rev. Lett. 42, 1008 (1979).
${ }^{150}$ D. Tillman, R. Thiel and E. Kisker, Z. Phys. B 77, 1 (1989).

${ }^{151}$ H. C. Siegmann, D. T. Pierce, and R. J. Celotta, Phys. Rev. Lett. 46, 452 (1981).

${ }^{152}$ M. Erbudak and N. Müller, Appl. Phys. Lett. 38, 575 (1981).

${ }^{153}$ M. Erbudak and G. Ravano, J. Appl. Phys. 52, 5032 (1981).

${ }^{154}$ D. T. Pierce, S. M. Girvin, J. Unguris, and R. J. Celotta, Rev. Sci. Instrum. 52, 1437 (1981), and references therein.

\section{PRICING AND ORDERING INFORMATION FOR REVIEW-ARTICLE REPRINTS}

PRICES: $\quad \$ 5.00 ; \$ 4.50$ each for bulk orders of ten or more copies of the same article sent to one address. Delivery is via surface mail. Airmail delivery available at the following surcharge: $\$ 2.50$ for the first copy plus $\$ 1.00$ for each additional copy sent to one address. Reprint orders must be prepaid.

ORDERS: Please specify REVIEW OF SCIENTIFIC INSTRUMENTS REVIEWS and give the article title, authors, month, and year of publication, and the page number of the article's title page. Send orders accompanied by payment in full (make checks payable to American Institute of Physics) to: Current Physics Reprints, American Institute of Physics, 335 East 45th Street, New York, NY 10017. 